\title{
MECP2 duplication and mutations impair NSCs differentiation via miR-197 regulated ADAM10
}

Yu-Meng Wang ${ }^{2,1, \#}$, Yu-Fang Zheng ${ }^{1,2,3, \#, *, ~ S i-Y u ~ Y a n g}{ }^{2,1}$, Zhang-Min Yang ${ }^{4}$, Lin-Na Zhang ${ }^{5}$, Yan-Qin

$\mathrm{He}^{4}$, Xiao-Hong Gong ${ }^{1}$, Dong Liu ${ }^{6}$, Richard H. Finnell ${ }^{7}$, Zi-Long Qiu ${ }^{8}$, Ya-Song Dü,*, Hong-Yan Wang ${ }^{1,3,9, *}$

1. Obstetrics and Gynecology Hospital, State Key Laboratory of Genetic Engineering at School of Life Sciences, Institute of Reproduction and Development, Fudan University, Shanghai 200011, China

2. Institute of Developmental Biology \& Molecular Medicine, Fudan University, Shanghai 200433, China

3. Key Laboratory of Reproduction Regulation of NPFPC, Collaborative Innovation Center of Genetics and Development, Fudan University, Shanghai 200032, China

4. Department of Biochemistry and Molecular Biology, College of life Sciences, Shaanxi Normal University, Xi'an 710062, China

5. Shanghai Mental Health Center, Shanghai Jiaotong University, Shanghai 200030, China

6. Co-innovation Center of Neuroregeneration, Jiangsu Key Laboratory of Neuroregeneration, Nantong University, Nantong, Jiangsu 226001, China

7. Departments of Molecular and Cellular Biology and Medicine, Baylor College of Medicine, Houston, Texas 77030, USA, and Collaborative Innovation Center for Genetics \& Development, School of Life Sciences, Fudan University, Shanghai 200438, China

8. Institute of Neuroscience, Shanghai Institutes for Biological Sciences, Chinese Academy of Sciences, Shanghai, 200031, China

9. Children's Hospital of Fudan University, 399 Wanyuan Road, Shanghai 201102, China

\# These authors contribute equally.

* Corresponding authors: Hongyan Wang: wanghy@fudan.edu.cn; Yasong Du: 13501942224@163.com; Yufang Zheng: zhengyf@fudan.edu.cn

Key words: MECP2, MDS, miR-197, ADAM10, neurogenesis

The authors have declared that no conflict of interest exists. 


\section{Abstract}

How MECP2 (Methyl-CpG-binding protein 2) duplication affects cortex development remains elusive. We found that elevated MeCP2 expression promotes neurogenesis during cortex development in $\operatorname{Tg}(M E C P 2)$ mouse brain. Ectopic expression of MeCP2 in NPCs inhibits ADAM10 and hence compromises the NOTCH pathway during NPC differentiation. MeCP2 up-regulates miR-197 to down-regulate ADAM10. The enhanced NPC differentiation/migration in Tg(MECP2) embryonic brain can be repressed by overexpression of ADAM10 or a miR-197 inhibitor.

Consistently, the reduced neurogenesis induced by three rare MeCP2 missense mutations (H371R, E394K, G428S) identified in a Han Chinese autism spectrum disorders (ASD) cohort, can be reversed by miR-197 both in vitro and in vivo. Our results revealed that a regulatory axis involving MeCP2, miR-197, ADAM10, and NOTCH signaling is critical for neurogenesis, which is affected by both MeCP2 duplication and mutation. 


\section{Introduction}

MECP2 (methyl-CpG binding protein 2), an X-linked gene encoding the methyl-cytosine binding protein MeCP2, is associated with two severe neurological disorders, Rett syndrome (RTT) and MECP2 duplication syndrome (MDS), which result from loss and gain of function of MECP2, respectively. Mutations in MECP2 were first identified as the monogenic cause for RTT (1). Although clinically distinguished from Autism Spectrum Disorder (ASD) by the Diagnostic and Statistical Manual of Mental Disorders (DSM-5) (2), autistic features are often observed (>60\%) in RTT patients $(3,4)$. The Mecp2 knockout mice (Mecp2-/y) also showed excellent replication/representation of RTT and ASD phenotypes $(5,6)$. Recently, MDS, a severe male intellectual disability syndrome, caused by MECP2 duplication was identified $(7,8)$, which has $~ 100 \%$ penetrance of autistic-like behaviors $(9$, 10). Transgenic mouse models for MDS $(11,12)$ and lentivirus-based transgenic monkey (13) both showed severe autistic-like and compulsive repetitive behaviors. Therefore, it has been suggested that the functional and dosage variations of MeCP2 are tightly associated with autistic features (4, 14). Exploring the functional conversions of MECP2 duplications and mutations may provide useful insight into the mechanisms underlying the development of ASD phenotypes $(4,14)$.

MeCP2 was originally identified as a transcriptional repressor $(15,16)$, but recent studies have shown it has diverse functions, including transcription activation (17), mRNA splicing (18, 19), and microRNA (miRNA) processing $(20,21)$, to name but a few. As both RTT and ASD patients show symptoms shortly after birth, most functional studies of MeCP2 have been focused on postnatal events such as dendritic arborization (22-24), synapse formation and plasticity (24-26), and adult neurogenesis (27-29). However, the function of MeCP2 during embryonic CNS development is still elusive. 
MeCP2 is widely and highly expressed in the developing central nervous system (CNS), including both the early neural tube and embryonic forebrain, in zebrafish, chicken, and mouse (30-33). However, embryonic development seems to be unaffected in Mecp2 knockout mice (6, 34). But study in Xenopus showed that MeCP2 promoted neurogenesis of Xenopus embryos, while the RTT mutant R168X failed to do so (35). A recent study in the monkey showed that Talent-edited mutation of MECP2 caused embryonic lethality in male mutant monkeys (36). Furthermore, human iPSCs generated from RTT patients with dysfunctional MeCP2 have abnormal neurogenesis and gliogenesis $(37,38)$. Therefore, it is likely that MeCP2 has an important role during early CNS development.

In the present study, we demonstrated that overexpressed MeCP2 promoted neurogenesis in the embryonic brain in the MDS model $\operatorname{Tg}(M E C P 2)$ mouse. Our results revealed a novel mechanism involving miR-197, ADAM10 (A disintergrin and metalloprotease 10), and NOTCH signaling as a critical regulatory axis for the enhanced neurogenesis induced by MeCP2. Furthermore, we identified three rare missense MECP2 mutations (H371R, E394K, G428S) in ASD patients in our Han Chinese ASD cohort, which are novel in the East Asian population according to ExAC (39). These MECP2 mutations resulted in dysfunctional regulation of miR-197 and neurogenesis. Surprisingly, while the inhibitor of miR-197 could reverse the effect of overexpressed MeCP2, the overexpression of miR-197 could reverse the NPCs differentiation defects caused by MECP2 mutations both in vitro and in vivo. Our results revealed a novel regulatory pathway via miR-197 by which MeCP2 acting on ADAM10/NOTCH signaling, implicating that molecules in this pathway are important for the etiology of MDS and possibly for ASD.

\section{Results}




\section{Neurogenesis is enhanced in $\mathrm{Tg}(M E C P 2)$ mouse fetal brain}

To determine the effect of $M E C P 2$ duplication on cell fate in vivo in fetal brain, we investigated a $\operatorname{Tg}(M E C P 2)$ mouse line which had previously been used as a model for MDS (11), as it contains an extra copy of human MECP2 and exhibits approximately doubled MeCP2 levels in the brain (Fig. 2D). During cortical neurogenesis in the mouse, NSCs/NPCs in the ventricular (VZ) and subventricular (SVZ) zones are differentiated into immature neurons and migrate radially to the cortical plate $(\mathrm{CP})$ to become mature neurons $(40,41)$. For quantification of cell fate in wild-type (WT) and $\operatorname{Tg}(M E C P 2)$ cortex, immunofluorescent staining on E18.5 and P7 brain sections with different markers were performed. The results revealed that there are significantly more Satb2+ (a cortical neuron marker) cells in CP layer and significantly less Sox $2^{+}$and $\mathrm{Tbr}^{+}$(progenitor markers) cells in VZ/SVZ layer of E18.5 Tg(MECP2) mice compared to WT littermates (Fig. 1A). By P7, there are still significantly more Satb2 $^{+}$and Tbr1 ${ }^{+}$neurons present in P7 Tg(MECP2) mice cortex than WT cortex (Fig. 1B).

Primary NPCs were isolated from E12.5 mouse cortex to further examine the in vitro effect of MeCP2 overexpression on NPCs differentiation. WT NPCs from C57BL/6 mice were infected with MeCP2 lentivirus after seeding on the culture dish. The exogenous expression doubled the expression levels of MeCP2 in those NPCs (Fig. 2A). Both transiently overexpressed MeCP2 in C57BL/6 NPCs and elevated MeCP2 in transgenic $\operatorname{Tg}(M E C P 2)$ mice significantly up-regulated the level of the neuronal marker MAP2, and down-regulated the level of the glia marker GFAP in NPCs (Fig. 2C\&D). Immunofluorescent staining on cultured NPCs infected by lentivirus also showed significantly more $\mathrm{MAP}^{+}$and less $\mathrm{GFAP}^{+}$cells with MeCP2 overexpression (Fig. 2B), which was consistent with previous reports (42). Taken together, the results demonstrate that elevated MeCP2 expression affects NPCs cell fate and promotes neurogenesis. 


\section{ADAM10 is downstream of MeCP2 in the control of neurogenesis}

Since NOTCH is a key molecule for NSCs fate $(43,44)$, we next examined the levels of several key molecules in the NOTCH pathway in primary cultured NPCs after overexpressing MeCP2. The levels of two NOTCH ligands JAG1 and DLL1, the full-length receptor NOTCH1, the activated $\mathrm{NOTCH}$ intracellular domain (NICD), and the two rate-limiting S2 enzymes for NOTCH cleavage, ADAM10 and ADAM17, were examined by Western blot analysis in primary NPCs transfected with either MeCP2 or an empty control vector. The results showed that the levels of NICD and ADAM10 were significantly down-regulated by MeCP2 expression in NPCs (Fig. 3A), while ADAM17, full-length NOTCH1, JAG1, and DLL1 were not significantly affected (Fig. S1A-E). These results suggest that MeCP2 might inhibit $\mathrm{NOTCH}$ signaling through the down-regulation of ADAM10, in order to regulate NPC development.

To study the relationship between ADAM10 and MeCP2, we expressed ADAM10 together with MeCP2 either in primary cultured NPCs or in embryonic brain. Expression of ADAM10 together with MeCP2 in NPCs reversed the effect of MeCP2 as indicated by the restoration of MAP2 and GFAP levels close to those of controls (Fig. 3B). The in vivo effect of ADAM10 on MeCP2 promoted neurogenesis was also tested in the developing cortex by in utero electroporation (IUE) experiments. Mouse embryonic brain were electroporated at embryonic day 14.5 (E14.5) and inspected at E18.5. There were significantly more $\mathrm{EYFP}^{+}$cells in CP layer in the $\mathrm{Tg}(\mathrm{MECP}$ ) cortex (Fig. 3D) as well as in C57BL/6 WT cortex ectopically overexpressing MeCP2 (Fig. 3C). Further, immunofluorescent staining showed that those $\mathrm{EYFP}^{+}$cells migrated into the CP layer were positive for the neuronal markers NeuN and Tuj1; whereas those cells remaining in the VZ/SVZ layer were positive for the progenitor markers Sox2 and Tbr2 (Fig. S2). When ADAM10 was co-expressed with MeCP2, it could 
reverse the effect of MeCP2 on neurogenesis, resulting in significantly fewer cells reaching the upper CP layer, compared to MeCP2 overexpression cortex (Fig. 3C\&D). Such effects were not observed when ADAM17 was co-expressed with MeCP2 (Fig. S1F\&G). These results suggest that ADAM10 is functionally downstream of MeCP2 during neurogenesis.

\section{MeCP2 down-regulates ADAM10 expression through up-regulating miR-197}

We next investigated how MeCP2 regulates ADAM10. Initially, we confirmed the regulation of ADAM10 by MeCP2 in human cells. Similar to cultured mouse NPCs, the ADAM10 protein level was also significantly down-regulated ( 53\%) by MeCP2 overexpression in a human glioblastoma cell line U251 (Fig. 4A). The mRNA level of ADAM10 was down-regulated $\sim 19 \%$ by MeCP2 (Fig. 4B). Since ADAM10 was not identified as a transcriptional target of MeCP2 (17), we screened the potential microRNAs (miRNAs) that might be involved in ADAM10 regulation by MeCP2. Seven miRNAs, identified as the most significantly regulated miRNAs by MeCP2 in the adult NSCs of the Mecp2-/y mouse (45), were used for our studies. When inhibitors for each miRNA (e.g. i-197 as an inhibitor of miR-197) were transfected into U251 cells, ADAM10 protein levels were significantly up-regulated by i-222, i-193 and i-197; while the other four inhibitors had no statistically significant effect (Fig. S3A). However, when MeCP2 was co-transfected with miRNA inhibitors, only i-197 and i-193 were able to reverse the MeCP2-induced down-regulation of ADAM10 protein (Fig. 4D \& Fig. S3B). The expression profile from miRNAMap shows that hsa-miR-193 is primarily expressed in the muscle, and hsa-miR-197 is highly expressed in the brain (http://mirnamap.mbc.nctu.edu.tw) (46); therefore, miR-197 is the most likely target of MeCP2 in the regulation of ADAM10 expression in the brain.

We went on to investigate the potential target site on ADAM10 3'-UTR for miR-197. A 7mer-m8 
miR-197 binding site at position 1568-1574 of human ADAM10 3'-UTR was predicted by TargetscanHuman (http://www.targetscan.org/vert_71/) (47), but it is poorly conserved between human and mouse (position 1563-1568 of mouse Adam10 3'-UTR) (Fig. 4E). A luciferase reporter A10-I-WT was constructed with a 305bp fragment of human ADAM10 3'-UTR covering this predicted position 1568-1574. The $G$ to A point mutation in the seed sequence was also constructed as A10-I-Mut (Fig. 4E). Both constructs were transfected into U251 cells with either miR-197 mimics or mimic control. Surprisingly, the results showed that miR-197 had no effect on either A10-I-WT nor A10-I-Mut (Fig. 4G), rather the point mutation blocked the interaction of human ADAM10 3'-UTR with miR-224, which has an overlapped seed sequence at position 1571-1577 (Fig. S4A\&B).

By using RNAhybrid analysis (48), a different miR-197 binding site at position 186-201 of human ADAM103'-UTR was predicted based on the free energy of the miRNA-target-duplex, which is highly conserved between human and mouse (position 185-199 of mouse Adam10 3'-UTR) (Fig. 4F). This binding site is different from traditional miRNAs and it was predicted to bind the 3' side of miR-197. A luciferase reporter A10-II-WT was constructed with a 402bp fragment of human ADAM10 3'-UTR covering positions 186-201. A deletion mutant reporter A10-II-Mut, deleting this untraditional miRNA binding site, was also constructed (Fig. 4F). The relative luciferase level was up-regulated approximately $23 \%$ by A10-II-Mut compared to that of A10-II-WT (Fig. 4G). Interestingly, miR-197 mimics significantly down-regulated the relative luciferase level of A10-II-WT but not A10-II-Mut (Fig. 4G). These results suggested a non-canonical interaction of miR-197 to ADAM10 3'-UTR. In support of this, an RNA-IP assay with a biotinylated miR-197 demonstrated that human ADAM10 3'-UTR could be pulled-down by miR-197 (Fig. 4H), which confirmed the interaction between miR-197 and ADAM10 3'-UTR. 
We subsequently sought to determine how MeCP2 regulates miR-197. RT-PCR experiments showed that the levels of pre- and matured miR-197 were significantly up-regulated by approximately 5.4 and 2.7 fold by MeCP2, respectively (Fig. 4J-K), while the level of pri-miR-197 was not affected by MeCP2 (Fig. 3l). This up-regulation of pre-miR-197 by MeCP2 does not affect the down-regulation of pre-miR-134 by MeCP2 (Fig. 4L). Taken together, MeCP2 down-regulated ADAM10 by up-regulating pre-miR-197 (Fig. 4M).

\section{MiR-197 plays a critical role in MeCP2 mediated neurogenesis}

Since MeCP2 expression is capable of up-regulating the expression of miR-197, we overexpressed miR-197 in primary cultured NPCs to better understand its effect on NPCs differentiation. Immunofluorescent staining showed that, like MeCP2, miR-197 overexpression promoted neurogenesis and inhibited gliogenesis, as evidenced by an elevated ratio of MAP2 ${ }^{+}$and decreased ratio of GFAP $^{+}$cells in the differentiated NPCs (Fig. 5A). The inhibitor of miR-197 (i-197) had opposite effect with decreased ratio of $\mathrm{MAP2}^{+}$and elevated ratio of $\mathrm{GFAP}^{+}$cells (Fig. 5A). Furthermore, the neurogenesis promoting effect of MeCP2 could also be blocked by i-197 when they were co-expressed in NPCs (Fig. 5B). MeCP2 induced down-regulation of ADAM10 was also blocked by i-197 (Fig. S5A).

Similar results were observed in the mouse fetal brain. The effect of overexpressing miR-197 alone was almost identical to MeCP2 overexpression in WT C57BL/6 mouse cortex, with more $\mathrm{EYFP}^{+}$cells reached the upper CP layer and less EYFP+ ${ }^{+}$cells stayed in the VZ/SVZ layer than in the controls (Fig. 5C). More importantly, i-197 blocked the effect caused by MeCP2 overexpression in WT brain. That is, the number of EYFP+ cells reaching the upper CP layer was reduced by i-197 (Fig. $5 C)$. Expression of $\mathrm{i}-197$ in $\operatorname{Tg}(M E C P 2)$ mice could also reverse the effect of elevated MeCP2 
expression, as significantly more $\mathrm{EYFP}^{+}$cells remained in the VZ/SVZ and IZ layers and fewer $\mathrm{EYFP}^{+}$cells reached the upper CP layer (Fig. S5B). These data strongly suggest that miR-197 plays a key role in MeCP2 mediated neurogenesis during cortical development.

\section{MECP2 mutations identified in a Chinese ASD cohort failed to promote neurogenesis}

A targeted-sequencing of MECP2 gene exons was performed in a Han Chinese cohort consisting of 288 ASD patients and 369 controls. Five rare missense MECP2 mutations were identified in six male ASD patients that were not observed among any of our controls (Table 1). These mutations were c.590C>T, c. $695 \mathrm{G}>\mathrm{C}, \quad$ c. $1112 \mathrm{~A}>\mathrm{G}, \quad$ c. $1180 \mathrm{G}>\mathrm{A}$, and c.1282G $>A$, which correlated to T197M, G232A, H371R, E394K and G428S in the human MeCP2-e1 protein (mouse MeCP2-e2) (Fig. S6), represents the isoform highly expressed in the brain $(32,49)$. The patients carrying these mutations showed a spectrum of symptoms, and the patient carrying the G428S mutation presented the most severe symptoms including severe intellectual disability with no functional language skills, and other medical conditions such as sleep disturbances (Table 1). According to ExAC, three C-terminal mutations (H371R, E394K and G428S) were not previously identified in the East Asian population (Total 4327 samples, with Male/Female=2,016/2,311) (39). The recurrent mutation $\mathrm{H} 371 \mathrm{R}$ in our cohort represents a novel mutation in all ExAC populations, a database which currently contains sixty thousand individuals (39). Therefore, we focused on those C-terminal mutations in the follow up studies.

We investigated the effect of three C-terminal mutants on NPCs differentiation. Although these mutations did not affect the expression levels of MeCP2 when overexpressed in NPCs (Fig. S7), they failed to promote neurogenesis in both NPCs and mouse embryonic cortex (Fig. 6B\&C). Both $\mathrm{MeCP} 2^{\mathrm{H} 371 \mathrm{R}}$ and MeCP2 ${ }^{\mathrm{E} 394 \mathrm{~K}}$ showed loss-of-function effects similar to that of MeCP2 ${ }^{380 \mathrm{x}}$, a 
previously reported loss-of-function truncation mutant (20) in IUE experiments, as they resulted in fewer EYFP+ cells reaching the upper CP layer, and more EYFP+ ${ }^{+}$cells remaining in the VZ/SVZ layer compared to the WT MeCP2 electroporated brain (Fig. 6B). To be noticed, MeCP2 ${ }^{\mathrm{G} 428 \mathrm{~S}}$ produced a more severe phenotype when overexpressed in mouse embryonic cortex with most of the MeCP2 ${ }^{\mathrm{G} 428 \mathrm{~S}}$ overexpressing cells remaining within the VZ/SVZ layers in the mouse cerebral cortex, and very few cells reached the CP at E18.5 (Fig. 6B).

Since the patient with the MeCP2 ${ }^{\mathrm{G} 428 \mathrm{~S}}$ mutation presented the most severe symptoms compared to other two mutations, and the MeCP2 ${ }^{\mathrm{G} 428 \mathrm{~S}}$ mutant had the strongest effect in IUE experiment, we went on to inspect the effects of MeCP2 ${ }^{\mathrm{G} 428 S}$ mutation in the in vitro NPC differentiation assay. Overexpression of the $M \mathrm{MCP}^{\mathrm{G} 428 \mathrm{~S}}$ mutant led to a significantly reduced percentage of $\mathrm{MAP}^{+}$cells $(\sim 21 \%)$ and a higher percentage of Nestin ${ }^{+}$cells $(\sim 42 \%)$, compared to either WT MeCP2 ( 87\% MAP2 ${ }^{+}$and $~ 15 \%$ Nestin $^{+}$cells, respectively) or the control empty vectors ( 49\% MAP2 $^{+}$and $\sim 22 \%$ Nestin $^{+}$cells, respectively) (Fig. 6C). The MeCP2G428S mutant demonstrated a gliogenesis promoting effect during NPC differentiation as indicated by a higher percentage of $\mathrm{GFAP}^{+}$cells ( 51\%) than WT MeCP2 ( 11\%), or the control ( 33\%) (Fig. 6C).

\section{MiR-197 reversed the neurogenesis defects caused by ASD-related MeCP2 mutants}

We also investigated the effect of those MeCP2 mutants on the levels of miR-197 and ADAM10. The results showed that three MeCP2 C-terminal mutants lost the ability to up-regulate pre-miR-197 and matured miR-197 (Fig. 7A\&B), or to down-regulate ADAM10 and NICD expression (Fig. S8). Again, MeCP2 ${ }^{\mathrm{G} 428 \mathrm{~S}}$ showed some gain-of-function effect evidenced as a significant up-regulation in NICD level in both mouse NPCs and human U251 cells (Fig. S8). However, when miR-197 was co-transfected with those three MeCP2 mutants, it was able to down-regulate the expression of 
ADAM10 even in presence of the MeCP2 mutants (Fig. 7C). Finally, when miR-197 was co-electroporated with MeCP2 ${ }^{\mathrm{G} 428 \mathrm{~S}}$ into E14.5 WT mouse brains, the abnormal distribution of cells detected in MeCP2 ${ }^{\mathrm{G} 428 \mathrm{~S}}$ expressing cortex at E18.5 could be almost completely reversed by exogenous miR-197 (Fig. 7D).

\section{Discussion}

Duplication or mutations of the MECP2 gene has been shown to cause MDS and RTT, respectively. $100 \%$ of MDS patients and $>60 \%$ of RTT patients express autistic behaviors, yet the underlying mechanism remains elusive. It has been proposed that altered NSC/NPC differentiation may contribute to the etiology of ASD (50). Again, the mechanism by which MeCP2 affects NSC/NPC differentiation is unknown. In this study, we demonstrated that the dosage and function of MeCP2 is tightly related to embryonic NSC/NPC differentiation. We showed in a MDS animal model that elevated MeCP2 expression promotes neurogenesis by down-regulating ADAM10 and suppressing $\mathrm{NOTCH}$ signaling. Moreover, we identified three rare missense mutations in MECP2 in an ASD cohort and found that they are loss-of-function mutants involved in regulating NSC/NPC differentiation. Our results revealed that the defects in a regulatory axis involving MeCP2, miR-197, ADAM10, and NOTCH signaling is associated with the etiology of MDS and/or ASD. Not only can ADAM10 and/or the miR-197 inhibitor inhibit the neurogenesis promoting effect of elevated MeCP2 levels in the $\operatorname{Tg}(M E C P 2)$ mouse brain, but additionally miR-197 can reverse the differentiation defects caused by the ASD-related MeCP2 mutants both in vitro and in vivo. These results indicate that miR-197 must be a critical molecule for the etiology of MDS and ASD.

The role of MeCP2 in NSC/NPC cell fate determination is complex and varies by its dosage, spatial, and temporal pattern. For example, there are studies reporting that Mecp2 deficiency does 
not affect NPCs fate in the Mecp2-/y mouse embryonic cortex $(51,52)$, rather it affects the neuron dendritic arborization and complexity in the mouse brain (51-53). However, overexpression of MeCP2 not only promotes neurogenesis of NPCs isolated from embryonic cortex (42), but also causes premature neurogenesis in the developing chick neural tube (33). Herein, we also observed enhanced neurogenesis in $\operatorname{Tg}(M E C P 2)$ mouse and in WT embryonic cortex with ectopic expression of MeCP2. Taken together, MECP2 duplication probably has more severe effects during the early embryonic stages. These observations are consistent with the clinical features of MDS patients, who have severe phenotypes starting from very early infantile stages $(9,10)$.

A surprising finding is that miR-197, which we identified as a crucial molecule downstream of MeCP2, does not regulate human ADAM10-3'UTR through the conventional miRNA seed sequence. Rather, miR-197 binds to an unconventional site on $A D A M 10-3^{\prime} \mathrm{UTR}$. The fact that this unconventional binding site but not the predicted miR-197 seed sequence is conserved between human and mouse suggests that the interaction between miR-197 to ADAM10-3'UTR is more similar to a siRNA. Although the mouse miR-197 was removed from miRBase in 2014 based on the fact that "the sequence does not map in a stem-loop region of the genomic sequence or any known mouse transcript sequence"; however, we noticed that small RNA libraries were used to identify miR-197 in both human and mouse in the original paper by Landgraf et al. (54). Indeed, a small RNA could be PCR amplified from E18.5 mouse brain by using has-miR-197 specific primers (Fig. S4C\&D). It is possible that there might be a small RNA with a similar sequence and function that exists in the mouse brain, even though it does not fit with the typical stem-loop structure of miRNAs.

Another interesting observation is that pre-miR-197 is up-regulated by MeCP2. Previous studies have shown that MeCP2 can both down-regulate and up-regulate miRNAs $(20,21,55)$. 
Down-regulation on miRNA, such as pre-miR-134, is due to MeCP2's binding to DGCR8 to interfere with the assembly of Drosha/DGCR8 miRNA processing complex (20). Indeed, pre-miR-134 is still down-regulated by MeCP2 in our system (Fig. 3L). Therefore, the MeCP2 regulatory mechanisms on miR-197 and miR-134 probably are unique to each miRNA. Recently, Tsujimura et al. found that miR-199a is up-regulated by MeCP2 through binding to Drosha and DDX5 (21). It is of interest that both pri-miR-199a (21) and pri-miR-197, but not pri-miR-134, can be experimentally pulled down by MeCP2 (Fig. S9). It is possible that when MeCP2 binds to a pri-miRNA, it facilitate the miRNA processing, but when MeCP2 cannot bind to a pri-miRNA, it interferes miRNA processing. Future studies to investigate whether other miRNAs regulated by MeCP2 also fit such rules are critically important.

It has been proposed that rare inherited mutations are important in the etiology of ASD (56-60). Indeed, most of the ASD cases with the MECP2 mutations in our cohort inherited the mutation from their asymptomatic carrier mother (Table 1). As all those ASD patients are boys, mutated MECP2 on the $\mathrm{X}$ chromosome would affect them more severely than their mothers. Although we cannot conclude that those mutations are causative without further genetic analysis of their whole genome, it is noticeable that the H371R mutation has two recurrent cases in our ASD patients (2/288), but does not exists in 61,075 controls from both ExAC and our cohort. Taken together with the fact that H371R has similar in vivo and in vitro effects as does the truncation mutation $380 \mathrm{X}$, it is highly possible that $\mathrm{H} 371 \mathrm{R}$ is a loss-of-function mutation with a high likelihood of being causative for ASD. The G428S mutation showed more severe effects on NPCs differentiation when compared to the 380X mutation. How this mutation changes MeCP2's function will need further investigation. One possibility is the G428 to S mutation might induce a new phosphorylation site on MeCP2, as previous studies have 
shown that the phosphorylation status of MeCP2 affects its functions $(28,61,62)$. There are 4 hemizygotes (male carriers) for G428S in the ExAC database from South Asian and Latino populations. However, the different genetic background of each population could affect the prevalence and susceptibility of any given mutation. If we look at only the East Asian population in ExAC, all three C-terminal mutations are novel out of a total of 4696 East Asian genomes. Whole-exome sequencing or even whole genome sequencing of our ASD cohort in combination with other ASD sequencing data sets are likely to be needed in the future to fully understand the genetic importance of those MECP2 mutations.

In conclusion, we uncovered a novel mechanism by which MeCP2 regulates NSC/NPC differentiation through the NOTCH pathway via miR-197. Together with a recent whole-exome sequencing study of over 1000 ASD cases (63) and our observations, deregulation of the NOTCH pathway could be important to the etiology of MDS and ASD.

\section{Material \& Methods}

\section{Animal Housing and Genotyping}

Both C57BL/6 mice and FVB-Tg(MECP2)1Hzo/J mice were maintained in the animal facility at the Institute of Developmental Biology \& Molecular Medicine, Fudan University. The protocol was approved by the Committee on the Ethics of Animal Experiments of Fudan University. The genotyping of FVB-Tg(MECP2) $1 \mathrm{Hzo} / \mathrm{J}$ mice were determined by PCR following the protocols from Jackson Laboratory website.

\section{Analyses of mouse cortical neurogenesis in vivo}

For quantification of cell fate in WT and FVB-Tg(MECP2) $1 \mathrm{Hzo} / \mathrm{J}$ mice at E18.5 and P7, the regions of the primary somatosensory cortex were identified and the numbers of Sox + , Tbr2+, 
Satb2+ or Tbr1+ cells were counted in each vertical column with $100 \mu \mathrm{m}$ width. All quantifications were performed with 4 brain sections from at least 3 animals. Data are presented as the mean \pm SEM and statistical significance was assessed using unpaired Student's t test.

\section{Primary mouse NPCs isolation and differentiation assay}

NPCs were isolated from E12.5 C57BL/6 or FVB mouse embryonic cortex. NPCs isolation and culture methods are based on previous reports $(42,64)$. NPCs were either transfected with X-tremeGENE HP DNA transfection reagent (Roche, 06366236001) or infected with different lentivirus (Obio Technology (Shanghai) Corp., Ltd.). 72 hours post-transfection or infection, NPCs cells were either lysed for western blot or subjected to immunofluorescent staining and imaging with a Zeiss LSM700 microscope. All films from western blot were scanned and analyzed with Quantity ONE based on intensity or were directly measured with Tanon gel image software. The results were normalized to its corresponding loading control GAPDH.

\section{In utero electroporation and cell counting}

In utero electroporation was performed as previously described $(65,66)$. About $1.5 \mu$ l of DNA mix was injected into each embryo. The ratio of either expressing plasmid or empty vector to pEYFP was 6:1. The ratio of (miR-197 inhibitor or ADAM10): (WT MeCP2): pEYFP was 3:3:1. The ratio of miR-197, MeCP2 ${ }^{\mathrm{G} 428 \mathrm{~S}}$, and pEYFP was 3:3:1. After electroporation and recovery, E18.5 embryos were collected and sectioned at $20 \mu \mathrm{m}$ and processed for further immunofluorescent analyses. Nuclear cell staining with DAPI was used to define different sub-regions of the cerebral cortex based on cell density, as previously described (65). The percentage of $\mathrm{EYFP}^{+}$cells in each layer was calculated based on total number of $\mathrm{EYFP}^{+}$cells in the same brain section. At least three sections from one brain were collected and at least four different embryos obtained from 3-4 different 
pregnant dams were collected for each group for statistical analyses. .

\section{Plasmids and Lentivirus}

Human MECP2-e1 cDNA was purchased from Origene. Point mutations were generated by mutagenesis PCR. The lentivrus for human WT MeCP2, H371K, G428S, 380X, sh-MeCP2 and sh-control were all purchased from Obio Technology (Shanghai) Corp., Ltd. The corresponding mutations were also constructed into rat MECP2-e2 cDNA with a HA tag in pRK5 vector and used in IUE.

Two different regions of human ADAM10 3'-UTR were cloned into Xbal/Fsel sites of pGL3 plasmid to generate luciferase reporter constructs. A10-I-WT contains 1461-1765 of the ADAM10 3'-UTR, which covers the predicted miR-197 binding site (1568-1574) by TargetscanHuman (http://www.targetscan.org/vert_71/) (47). A10-II-WT contains 4-405 of human ADAM10 3'-UTR, which covers the predicted binding site (186-201) by RNAhybrid analysis (48).

\section{qRT-PCR}

Total RNA from NPCs or U251 was extracted with RNAeasy kit (QIAGEN, Cat\# 74104). The levels of ADAM10 mRNA were determined using the qRT-PCR kit (Takara RR036A and RR820A) with GAPDH as internal control. The levels of mature miR-197 were determined with All-in-One ${ }^{\mathrm{TM}}$ miRNA qRT-PCR Detection Kit (GeneCopoeia, QP015). The levels of pri-miR-197 were detected with TaqMan pri-microRNA assay kit (Applied Biosystems) following reverse transcript with Toyobo ReverTra Ace- $\alpha$ kit (Toyobo). The levels of pre-miR-197 were detected with miScript Precusor Assay (QIAGEN) following reverse transcript with miScript II RT Kit (QIAGEN).

\section{RNA Immunoprecipitation}

U251 cells were transfected with empty vector, WT MeCP2, or ASD-related MeCP2 mutant 
expressing plasmids. 24 hours later, cells were collected for cross-linking, lysing, and sonication and were subjected to the RNA immunoprecipitation assay using previously described protocols $(21,67)$. Anti-MeCP2 antibody (ab2828) and rabbit immunoglobulin G (IgG) antibodies were used. The immunoprecipitated RNA was analyzed by qRT-PCR described before.

\section{Biotinylated Micro-RNA Pull Down Assay for Identifying miRNA Targets}

Biotinylated double stranded miRNA-197 and its scrambled control miRNA (B02003) (both Biotin-labeled at 3' end) were purchased from GenePharma. U251 cells were transfected with control miRNA and miR-197 at a final concentration of $100 \mathrm{nM}$ with RNAimax (Invitrogen $\left.{ }^{\mathrm{TM}}, 13778150\right) .24$ hours post transfection, whole cell lysates were harvested and subjected to RNP pull down followed with RT-PCR. The miRNA enrichment was calculated as follow: Bi-miR-197 pull-down for ADAM10 3'UTR /Scramble control pull-down for ADAM10 3'UTR = X, Bi-miR-197 input/Bio-Scramble control input $=\mathrm{Y}$, Fold binding $=\mathrm{X} / \mathrm{Y}$. At least three independent experiments with a minimum of three replicates each time were performed for each set.

\section{Statistical analyses}

All experiments were repeated at least three times and the statistical significance was evaluated. Data are expressed as mean \pm SEM. Statistical differences were calculated by two-tailed unpaired $t$ test for two datasets and ANOVA followed by Bonferroni post hoc test for multiple datasets using Prism (GraphPad Inc., La Jolla, CA). p<0.05 was considered statistically significant.

\section{Human subjects}

Blood samples from 288 ASD patients (mean age $6.1 \pm 3.1$ years, $85 \%$ male) were collected between 2007 and 2010 at the Shanghai Mental Health Center of Shanghai Jiaotong University. Protocols were reviewed and approved by the Ethics Committee of Fudan University and Shanghai 
Jiaotong University prior to the commencement of the study. Written informed consent from the parents or guardians of the children was obtained prior to inclusion in the study. The 369 controls (mean age 19 years, 81\% male) were unrelated healthy volunteers from the freshman student class at Fudan University (2010), which were ethnically and gender-matched from the same geographical area. DNA was extracted and all samples were pooled together for targeted sequencing on the $2 \mathrm{~kb}$ upstream, 5'-UTR, 3'-UTR, and coding regions of MECP2 gene was carried out at GBP Biotechnology (Jiangsu, China). Sanger sequencing was performed to confirm those 5 missense mutations in the 6 ASD cases (Fig. S8A).

\section{Acknowledgement}

We would like to thank Dr. Xiongli Yang, Dr. Xiang Yu, and Dr. Xiaohui Wu for their valuable suggestions on our manuscript.

This work was supported by the following grants to Drs. Hongyan Wang and Yufang Zheng: the National Key Basic Research Program of China (2016YFC1000502), the NSFC (81430005, 31771669, 81741048, 31521003), the National Basic Research Program (973) of China (2013CB945404), the Commission for Science and Technology of Shanghai Municipality (17JC1400902 and 14JC1401000), the 863 program of China (2014AA021104); and the Changjiang Scholarship to Dr. Richard H. Finnell. 


\section{Reference}

1. Amir RE, et al. (1999) Rett syndrome is caused by mutations in X-linked MECP2, encoding methyl-CpG-binding protein 2. Nat Genet 23(2):185-188.

2. American Psychiatric Association. \& American Psychiatric Association. DSM-5 Task Force. (2013) Diagnostic and statistical manual of mental disorders : DSM-5 (American Psychiatric Association, Arlington, VA) 5th Ed pp xliv, $947 \mathrm{p}$.

3. Neul JL, et al. (2008) Specific mutations in methyl-CpG-binding protein 2 confer different severity in Rett syndrome. Neurology 70(16):1313-1321.

4. Percy AK (2011) Rett syndrome: exploring the autism link. Archives of neurology 68(8):985-989.

5. Chao HT, et al. (2010) Dysfunction in GABA signalling mediates autism-like stereotypies and Rett syndrome phenotypes. Nature 468(7321):263-269.

6. Guy J, Hendrich B, Holmes M, Martin JE, \& Bird A (2001) A mouse Mecp2-null mutation causes neurological symptoms that mimic Rett syndrome. Nat Genet 27(3):322-326.

7. Van Esch H, et al. (2005) Duplication of the MECP2 region is a frequent cause of severe mental retardation and progressive neurological symptoms in males. Am J Hum Genet 77(3):442-453.

8. Samaco RC, et al. (2008) A partial loss of function allele of methyl-CpG-binding protein 2 predicts a human neurodevelopmental syndrome. Hum Mol Genet 17(12):1718-1727.

9. Ramocki MB, Tavyev YJ, \& Peters SU (2010) The MECP2 duplication syndrome. American journal of medical genetics. Part A 152A(5):1079-1088.

10. Ramocki MB, et al. (2009) Autism and other neuropsychiatric symptoms are prevalent in individuals with MeCP2 duplication syndrome. Ann Neurol 66(6):771-782.

11. Collins AL, et al. (2004) Mild overexpression of MeCP2 causes a progressive neurological disorder in mice. Hum Mol Genet 13(21):2679-2689.

12. Sztainberg Y, et al. (2015) Reversal of phenotypes in MECP2 duplication mice using genetic rescue or antisense oligonucleotides. Nature 528(7580):123-126.

13. Liu Z, et al. (2016) Autism-like behaviours and germline transmission in transgenic monkeys overexpressing MeCP2. Nature.

14. Lombardi LM, Baker SA, \& Zoghbi HY (2015) MECP2 disorders: from the clinic to mice and back. J Clin Invest 125(8):2914-2923.

15. Jones PL, et al. (1998) Methylated DNA and MeCP2 recruit histone deacetylase to repress transcription. Nat Genet 19(2):187-191.

16. Nan X, et al. (1998) Transcriptional repression by the methyl-CpG-binding protein MeCP2 involves a histone deacetylase complex. Nature 393(6683):386-389.

17. Chahrour M, et al. (2008) MeCP2, a key contributor to neurological disease, activates and represses transcription. Science 320(5880):1224-1229.

18. Young Jl, et al. (2005) Regulation of RNA splicing by the methylation-dependent transcriptional repressor methyl-CpG binding protein 2. Proc Natl Acad Sci U S A 102(49):17551-17558.

19. Cheng TL, et al. (2017) Regulation of mRNA splicing by MeCP2 via epigenetic modifications in the brain. Sci Rep 7:42790.

20. Cheng TL, et al. (2014) MeCP2 suppresses nuclear microRNA processing and dendritic growth by regulating the DGCR8/Drosha complex. Dev Cell 28(5):547-560.

21. Tsujimura K, et al. (2015) miR-199a Links MeCP2 with mTOR Signaling and Its Dysregulation Leads to Rett Syndrome Phenotypes. Cell reports 12(11):1887-1901.

22. Kishi N \& Macklis JD (2010) MeCP2 functions largely cell-autonomously, but also non-cell-autonomously, in 
neuronal maturation and dendritic arborization of cortical pyramidal neurons. Exp Neurol 222(1):51-58.

23. Zhou Z, et al. (2006) Brain-specific phosphorylation of MeCP2 regulates activity-dependent Bdnf transcription, dendritic growth, and spine maturation. Neuron 52(2):255-269.

24. Guy J, Cheval H, Selfridge J, \& Bird A (2011) The role of MeCP2 in the brain. Annual review of cell and developmental biology 27:631-652.

25. Cheng J, et al. (2014) SUMOylation of MeCP2 is essential for transcriptional repression and hippocampal synapse development. J Neurochem 128(6):798-806.

26. Qiu S, Aldinger KA, \& Levitt P (2012) Modeling of autism genetic variations in mice: focusing on synaptic and microcircuit dysfunctions. Dev Neurosci 34(2-3):88-100.

27. Kohyama J, et al. (2008) Epigenetic regulation of neural cell differentiation plasticity in the adult mammalian brain. Proc Natl Acad Sci U S A 105(46):18012-18017.

28. Li H, et al. (2014) Cell cycle-linked MeCP2 phosphorylation modulates adult neurogenesis involving the Notch signalling pathway. Nat Commun 5:5601.

29. Chen Z, et al. (2017) Accumulated quiescent neural stem cells in adult hippocampus of the mouse model for the MECP2 duplication syndrome. Sci Rep 7:41701.

30. Gao H, et al. (2015) Mecp2 regulates neural cell differentiation by suppressing the Id1 to Her2 axis in zebrafish. J Cell Sci 128(12):2340-2350.

31. Nozawa K, et al. (2017) Zebrafish Mecp2 is required for proper axonal elongation of motor neurons and synapse formation. Developmental neurobiology.

32. Pelka GJ, Watson CM, Christodoulou J, \& Tam PP (2005) Distinct expression profiles of Mecp2 transcripts with different lengths of $3^{\prime}$ UTR in the brain and visceral organs during mouse development. Genomics 85(4):441-452.

33. Petazzi P, et al. (2014) An increase in MECP2 dosage impairs neural tube formation. Neurobiol Dis 67:49-56.

34. Chen RZ, Akbarian S, Tudor M, \& Jaenisch R (2001) Deficiency of methyl-CpG binding protein-2 in CNS neurons results in a Rett-like phenotype in mice. Nat Genet 27(3):327-331.

35. Stancheva I, Collins AL, Van den Veyver IB, Zoghbi H, \& Meehan RR (2003) A mutant form of MeCP2 protein associated with human Rett syndrome cannot be displaced from methylated DNA by notch in Xenopus embryos. Mol Cell 12(2):425-435.

36. Chen Y, et al. (2017) Modeling Rett Syndrome Using TALEN-Edited MECP2 Mutant Cynomolgus Monkeys. Cell 169(5):945-955 e910.

37. Andoh-Noda T, et al. (2015) Differentiation of multipotent neural stem cells derived from Rett syndrome patients is biased toward the astrocytic lineage. Molecular brain 8:31.

38. Mellios N, et al. (2017) MeCP2-regulated miRNAs control early human neurogenesis through differential effects on ERK and AKT signaling. Molecular psychiatry.

39. Lek M, et al. (2016) Analysis of protein-coding genetic variation in 60,706 humans. Nature 536(7616):285-291.

40. Borrell V \& Reillo I (2012) Emerging roles of neural stem cells in cerebral cortex development and evolution. Developmental neurobiology 72(7):955-971.

41. Kriegstein A \& Alvarez-Buylla A (2009) The glial nature of embryonic and adult neural stem cells. Annu Rev Neurosci 32:149-184.

42. Tsujimura K, Abematsu M, Kohyama J, Namihira M, \& Nakashima K (2009) Neuronal differentiation of neural precursor cells is promoted by the methyl-CpG-binding protein MeCP2. Exp Neurol 219(1):104-111.

43. Zhou ZD, Kumari U, Xiao ZC, \& Tan EK (2010) Notch as a molecular switch in neural stem cells. IUBMB Life 62(8):618-623.

44. Yoon K \& Gaiano N (2005) Notch signaling in the mammalian central nervous system: insights from mouse mutants. Nat Neurosci 8(6):709-715. 
45. Szulwach KE, et al. (2010) Cross talk between microRNA and epigenetic regulation in adult neurogenesis. J Cell Biol 189(1):127-141.

46. Hsu SD, et al. (2008) miRNAMap 2.0: genomic maps of microRNAs in metazoan genomes. Nucleic Acids Res 36(Database issue):D165-169.

47. Agarwal V, Bell GW, Nam JW, \& Bartel DP (2015) Predicting effective microRNA target sites in mammalian mRNAs. Elife 4.

48. Rehmsmeier M, Steffen P, Hochsmann M, \& Giegerich R (2004) Fast and effective prediction of microRNA/target duplexes. Rna 10(10):1507-1517.

49. Dragich JM, Kim YH, Arnold AP, \& Schanen NC (2007) Differential distribution of the MeCP2 splice variants in the postnatal mouse brain. J Comp Neurol 501(4):526-542.

50. Stevens HE, Smith KM, Rash BG, \& Vaccarino FM (2010) Neural stem cell regulation, fibroblast growth factors, and the developmental origins of neuropsychiatric disorders. Frontiers in neuroscience 4.

51. Kishi N \& Macklis JD (2004) MECP2 is progressively expressed in post-migratory neurons and is involved in neuronal maturation rather than cell fate decisions. Mol Cell Neurosci 27(3):306-321.

52. Kishi N, et al. (2016) Reduction of aberrant NF-kappaB signalling ameliorates Rett syndrome phenotypes in Mecp2-null mice. Nat Commun 7:10520.

53. Smrt RD, et al. (2007) Mecp2 deficiency leads to delayed maturation and altered gene expression in hippocampal neurons. Neurobiol Dis 27(1):77-89.

54. Landgraf $P$, et al. (2007) A mammalian microRNA expression atlas based on small RNA library sequencing. Cell 129(7):1401-1414.

55. Wu H, et al. (2010) Genome-wide analysis reveals methyl-CpG-binding protein 2-dependent regulation of microRNAs in a mouse model of Rett syndrome. Proc Natl Acad Sci U S A 107(42):18161-18166.

56. Iossifov I, et al. (2012) De novo gene disruptions in children on the autistic spectrum. Neuron 74(2):285-299.

57. Neale BM, et al. (2012) Patterns and rates of exonic de novo mutations in autism spectrum disorders. Nature 485(7397):242-245.

58. Sanders SJ, et al. (2012) De novo mutations revealed by whole-exome sequencing are strongly associated with autism. Nature 485(7397):237-241.

59. O'Roak BJ, et al. (2012) Sporadic autism exomes reveal a highly interconnected protein network of de novo mutations. Nature 485(7397):246-250.

60. Yuen RK, et al. (2015) Whole-genome sequencing of quartet families with autism spectrum disorder. Nat Med 21(2):185-191.

61. Cohen S, et al. (2011) Genome-wide activity-dependent MeCP2 phosphorylation regulates nervous system development and function. Neuron 72(1):72-85.

62. Ebert $\mathrm{DH}$, et al. (2013) Activity-dependent phosphorylation of MeCP2 threonine 308 regulates interaction with NCoR. Nature 499(7458):341-345.

63. Hormozdiari F, Penn O, Borenstein E, \& Eichler EE (2015) The discovery of integrated gene networks for autism and related disorders. Genome research 25(1):142-154.

64. Liu B, et al. (2016) MAZ mediates the cross-talk between CT-1 and NOTCH1 signaling during gliogenesis. Sci Rep $6: 21534$.

65. Li $\mathrm{Q}$, et al. (2013) ADAM17 is critical for multipolar exit and radial migration of neuronal intermediate progenitor cells in mice cerebral cortex. PLoS One 8(6):e65703.

66. Saito T (2006) In vivo electroporation in the embryonic mouse central nervous system. Nature protocols 1(3):1552-1558.

67. Niranjanakumari S, Lasda E, Brazas R, \& Garcia-Blanco MA (2002) Reversible cross-linking combined with immunoprecipitation to study RNA-protein interactions in vivo. Methods 26(2):182-190. 


\section{Figure legends}

Figure 1. MECP2 duplication promotes neurogenesis in $\mathrm{Tg}(M E C P 2)$ FVB mice cortex. Coronal brain sections from E18.5 (A) and P7 (B) Tg(MECP2) FVB mice or WT littermates were stained with cortical neuron marker Satb2, deep layer neuron marker Tbr1, and progenitor markers Sox2 and Tbr2. $\mathrm{N} \geq 4$. DAPI (blue) was used for nuclear staining. The numbers of positive cells within $100 \mu \mathrm{m}$ bin were counted. Representative sections are shown in left and statistical analysis are shown in right panels, respectively. All statistic data represent means \pm SEM. $* p<0.05, * * * p<0.001$. Scale bar is $50 \mu \mathrm{m}$ in $\mathrm{E} 18.5$ and $100 \mu \mathrm{m}$ in $\mathrm{P} 7$.

Figure 2. MECP2 duplication promotes neurogenesis of cultured NPCs. Mouse primary NPCs isolated from C57BL/6 mouse E12.5 embryonic cortex were infected with MeCP2 expressing lentivirus and cultured for $72 \mathrm{hrs}(\mathbf{A}-\mathbf{C})$. Cell lysates were subjected to western blotting or immunofluorescent staining. (A) Exogenous expressing of MeCP2 doubled its expression level in NPCs. GAPDH was used as loading control. $\mathrm{N}=3$. Representative blot is shown on the top panel and statistical analysis for MeCP2 levels are shown in the bottom panel. (B) Immunofluorescent staining for GFAP, MAP2, and NESTIN were performed on NPCs. N=9. The MeCP2 and control viruses contain EGFP and showed here as green. The staining for MAP2, GFAP, and NESTIN are labeled with pseudo-colored red. Representative images are shown on the left and the percentage of Nestin ${ }^{+}$, $\mathrm{MAP2}^{+}$and $\mathrm{GFAP}^{+}$cells within $\mathrm{EGFP}^{+}$cells are shown on the right panels respectively. Scale bar is $50 \mu \mathrm{m}$. (C) Western blot analysis for MAP2, GFAP, and HA labeled MeCP2. GAPDH was used as loading control. $\mathrm{N}=9$. Representative blot is shown on the left, and statistical analysis for MAP2 and GFAP levels are shown in the right panels, respectively. (D) Mouse primary NPCs isolated from FVB WT and $\operatorname{Tg}(M E C P 2)$ mouse E12.5 embryonic cortex were directly cultured for $72 \mathrm{hrs}$. Cell lysates were subjected to western blot analysis for both MeCP2 and differentiation markers, MAP2 and GFAP. GAPDH was used as loading control. $\mathrm{N}=3$. Representative blot is shown in left and statistical analysis for MeCP2, MAP2 and GFAP levels are shown in right panels, respectively. All statistic data represent means \pm SEM. $* p<0.05, * * p<0.01, * * * p<0.001$. 
Figure 3. ADAM10 is a critical down-stream molecule of MeCP2 in regulating NPCs differentiation. (A) Mouse primary NPCs isolated from E12.5 C57BL/6 mouse embryonic cortex were transfected with either empty vector or MeCP2 expressing plasmids and cultured for $72 \mathrm{hrs}$. Cell lysates were subjected to Western blot analysis for ADAM10 and NICD. GAPDH was used as a loading control. $\mathrm{N} \geq 5$. Representative blots are shown on the left and statistical analyses for ADAM10 and NICD were presented on the right panels. (B) Overexpression of ADAM10 together with WT MeCP2 could reverse the differentiation effects of MeCP2 in cultured NPCs. Mouse primary NPCs isolated from E12.5 C57BL/6 mouse embryonic cortex were transfected with HA tagged MeCP2 expressing plasmids with either control vector or ADAM10 expressing plasmid. 72hrs later, NPCs lysates were subjected to Western blot analysis for MAP2, GFAP, ADAM10, and HA. GAPDH was used as a loading control. $\mathrm{N} \geq 5$. Representative blots are shown on the left panel and statistical analyses for GFAP, MAP2, and ADAM10 were presented on the right panel, respectively. Overexpression of ADAM10 could reverse the differentiation effects of MeCP2 in electroporated fetal brains (C-D). (C) C57BL/6 mouse were electroporated at E14.5 with either MeCP2 or MeCP2 with ADAM10 at 1:1 ratio. All samples were collected at E18.5 for sectioning and immunostaining. Each condition was repeated in four different embryos from four pregnant female mice $(\mathrm{N}=4)$. Representative brain sections are presented on the left and the EYFP ${ }^{+}$cells in each layer were counted and compared to the total $\mathrm{EYFP}^{+}$cells. The statistical analyses for cells in each layer are shown on the right panel. (D) $T g(M E C P 2)$ or WT FVB mice cortex were electroporated with EYFP or ADAM10 with EYFP plasmid ( $N=4)$. Representative brain sections are presented on the left and the $\mathrm{EYFP}^{+}$cells in each layer were counted and compared to the total EYFP+ ${ }^{+}$cells. The statistical analyses for cells in each layer are presented on the right panel. DAPI (blue) was used for nuclear staining. Scale bar is $50 \mu \mathrm{m}$ in E18.5 and $100 \mu \mathrm{m}$ in P7. All statistic data represent means \pm SEM. *,\# $p<0.05, * *, \# \# p<0.01, * * *, \# \# \# p<0.001$.

Figure 4. MiR-197 is up-regulated by MeCP2 to down-regulate ADAM10. (A-B) Human glioblastoma cells U251 were transfected with empty control or plasmid to overexpress MeCP2, and subjected to either western blotting or qRT-PCR to detect ADAM10 protein (A) or mRNA (B). N $\geq 5$. (C-D) U251 cells were transfected with MeCP2 and a miR-197 inhibitor or scramble control, and subjected to qRT-PCR for ADAM10 mRNA (C) or Western blotting for ADAM10 protein (D). N $\geq 3$. 
GAPDH was used as loading control in both experiments. (E) A 7mer-m8 miR-197 binding site predicted by TargetscanHuman. The alignment of miR-197 to human and mouse ADAM10 3'-UTR and the point mutation $\mathrm{G}>\mathrm{A}$ in A10-I-Mut are illustrated. (F) A miR-197 binding site predicted by RNAhybrid is highly conserved between human and mouse. The deletion mutation in A10-II-Mut was also illustrated. (G) Luciferase reporter plasmids for ADAM10 3'-UTR were transfected into U251 cells with miR-197 mimics or control. $\mathrm{N} \geq 8$. (H) Biotin-labeled miR-197 and control scramble were transfected into U251 cells and the ADAM10 3'-UTR pulled down by miR-197 or control scramble were quantified by qRT-PCR. N=4. (I-L) U251 cells were transfected with either control empty vector or MeCP2 expressing plasmids and cultured for $24 \mathrm{hrs}$. RNA from these cells were extracted and subjected for qRT-PCR. N=9. The levels of pri-miR-197, pre-miR-197, mature miR-197, and pre-miR-134 were shown in (I-L), respectively. All statistical data are represented as means \pm SEM. * $p<0.05, * * p<0.01, * * * p<0.001$. (M) A scheme illustration for MeCP2 regulation on miR-197 and ADAM10.

Figure 5. miR-197 promotes neurogenesis and the inhibitor of miR-197 reverses the effect of MeCP2 overexpression. (A) Primary NPCs from C57BL/6 mouse were infected with assorted lentivirus to over-express either miR-197, miR-control, miR-197 inhibitor (i-197), or inhibitor-control. 72 hrs later, cells were subjected for immunofluorescent staining. The infected cells are labeled with pseudo-colored green. The staining for MAP2, GFAP, and NESTIN are labeled with pseudo-colored red. The percentage of $\mathrm{Nestin}^{+}, \mathrm{MAP}^{+}$and $\mathrm{GFAP}^{+}$cells within infected cells are shown on the right side respectively in. $\mathrm{N} \geq 4$. (B) Primary mouse NPCs were infected with assorted lentivirus to overexpress MeCP2 with either $\mathrm{i}-197$ or $\mathrm{i}$-cont. 72 hrs later, cells were subjected for immunofluorescent staining for MAP2, GFAP, and NESTIN. The MeCP2 and control viruses contain EGFP and showed here as green. The $\mathrm{i}-197 / \mathrm{i}-\mathrm{cont}$ viruses infected cells are pseudo-color labeled as blue. The staining for MAP2, GFAP, and NESTIN are labeled with pseudo-colored red. The percentage of $\mathrm{Nestin}^{+}, \mathrm{MAP}^{+}$and $\mathrm{GFAP}^{+}$cells within green and blue double positive cells (cyan in nucleus, white arrow head) are shown on the right side, respectively. $N=9$. (C) Overexpression of miR-197 mimicked the effect of MeCP2 and miR-197 inhibitors (i-197) blocked the effect of overexpressed MeCP2 in WT C57BL/6 mouse fetal brain by IUE experiments. $\mathrm{N}=9$. Representative brain sections are presented on the left side, and the statistical analyses for cells in each layer on the 
right panel. All statistical data are presented as means \pm SEM. * or \# $p<0.05$, ** or \#\# $p<0.01, * * *$ or $\# \# \#$ p $<0.001$. Scale bar is $50 \mu \mathrm{m}$.

Figure 6. ASD-related MeCP2 mutants lose the effect on promoting neurogenesis. (A) A scheme illustration for positions of the ASD-related MECP2 mutations, H371R, E394K, G428S, and a reported truncated mutant 380X. (B) Fetal WT C57BL/6 mouse brains were electroporated at E14.5 to overexpress either WT MeCP2 or ASD-related MeCP2 mutants. Samples were collected at E18.5 for sectioning and immunostaining. Each condition was repeated in four different embryos from four pregnant dams $(\mathrm{N}=4)$. Representative brain sections are presented on the left and the statistical analyses for cells in each layer are shown on the right. (C) Primary mouse NPCs were infected with assorted lentivirus to overexpress WT MeCP2, MeCP2 ${ }^{\mathrm{H} 371 \mathrm{R}}$, MeCP2 ${ }^{\mathrm{G} 428 \mathrm{~S}}$, and MeCP2 ${ }^{380 x} .72 \mathrm{hrs}$ later, cells were subjected for immunofluorescent staining for MAP2, GFAP, and NESTIN. The infected cells are labeled with EGFP (green). The staining for MAP2, GFAP, and NESTIN are labeled with pseudo-colored red. The percentage of $\mathrm{MAP2}^{+}$, $\mathrm{GFAP}^{+}$, and $\mathrm{Nestin}^{+}$cells within $\mathrm{EGFP}^{+}$cells are shown on the right, respectively. $\mathrm{N}=9$. All statistical data are presented as means \pm SEM. $*$ or \# $p<0.05, * *$ or \#\# $p<0.01, * * *$ or \#\#\# $p<0.001$. Scale bar is $50 \mu \mathrm{m}$.

Figure 7. MiR-197 reverses the neurogenesis defects induced by ASD-related MeCP2 mutants. U251 cells were transfected with either WT or mutant MeCP2 expressing plasmids and cultured for 24hrs. RNA from these cells was subjected to qPCR for either mature miR-197 (A), pre-miR-197 (B), or pri-miR-197 (C). $\mathrm{N}=6$ for mature miR-197, $\mathrm{N}=4$ for pre and pri-miR-197. (D) U251 cells were transfected with mutant MeCP2 expressing plasmids and either control or miR-197 plasmid. Cells were cultured for $24 \mathrm{hrs}$ and cell lysates were subjected to western blot for ADAM10. $\mathrm{N} \geq 5$. Representative blots are shown on the left side and statistical analyses are shown on the right side, respectively. (E) Overexpression of miR-197 reversed the differentiation defects caused by MeCP2 ${ }^{\mathrm{G} 428 \mathrm{~S}}$ in electroporated fetal brain. Each condition was repeated in four different embryos from four pregnant dams $(\mathrm{N}=4)$. Representative brain sections are presented on the left, and the statistical analyses for cells in each layer is shown on the right. All statistical data are presented as means \pm SEM. $*$ or $\# p<0.05, * *$ or \#\# $p<0.01, * * *$ or \#\#\# $p<0.001$. Scale bar is $50 \mu \mathrm{m}$. 
bioRxiv preprint doi: https://doi org/101101/312983; this version posted May 2, 2018. The copyright holder for this preprint (which was not certified by peer review) is the author/funder, who has granted bioRxiv a license to display the preprint in perpetuity. It is made available under aCC-BY 4.0 International license.

Table 1. Rare Mutations of MECP2 detected in Chinese ASD cohort

\begin{tabular}{|c|c|c|c|c|c|c|c|c|c|c|c|c|c|c|}
\hline cDNA & $\begin{array}{l}\text { Amino } \\
\text { acid }\end{array}$ & $\begin{array}{l}\text { Domain } \\
\text { location }\end{array}$ & Patient & $\begin{array}{l}\text { Birth } \\
\text { Year }\end{array}$ & Sex & $\begin{array}{l}\text { Intellectual } \\
\text { Disability }\end{array}$ & $\begin{array}{l}\text { Functional } \\
\text { language }\end{array}$ & $\begin{array}{c}\text { Other medical } \\
\text { issues }\end{array}$ & Mother & $\begin{array}{l}\text { AF in } \\
\text { ASD }^{a}\end{array}$ & $\begin{array}{l}\text { AF in } \\
\text { Cont }\end{array}$ & $A F$ in ExAC & $P$ value $^{d}$ & $\begin{array}{c}A F \text { in ExAC } \\
E A^{C}\end{array}$ \\
\hline $590 \mathrm{C}>\mathrm{T}$ & T197M & Interdomain & ASD164 & 2007 & M & NO & Yes & NO & Carrier & $\begin{array}{l}0.00303 \\
(1 / 330)\end{array}$ & 0 & $\begin{array}{l}0.0005475 \\
(48 / 87676)\end{array}$ & 0.169 & $\begin{array}{c}0.0004519 \\
(3 / 6638)\end{array}$ \\
\hline $695 \mathrm{G}>\mathrm{C}$ & G232A & TRD & ASD97 & 2002 & M & NO & Yes & ADHD & Carrier & $\begin{array}{l}0.00303 \\
(1 / 330)\end{array}$ & 0 & $\begin{array}{l}0.0001942 \\
(17 / 87526)\end{array}$ & 0.066 & $\begin{array}{l}0.00196 \\
(13 / 6634)\end{array}$ \\
\hline $1112 A>G$ & H371R & C-term & ASD348 & 2008 & M & Yes (mild) & NO & ADHD & $\begin{array}{l}\text { Carrier } \\
\text { Carrier }\end{array}$ & $\begin{array}{l}0.00606 \\
(2 / 330)\end{array}$ & 0 & 0 & $1.4 \mathrm{e}-05$ & 0 \\
\hline $1180 G>A$ & E394K & C-term & ASD243 & 2004 & M & $\begin{array}{c}\text { Yes } \\
\text { (moderate) }\end{array}$ & NO & $\begin{array}{c}\text { ADHD/mood } \\
\text { disorder }\end{array}$ & NA & $\begin{array}{l}0.00303 \\
(1 / 330)\end{array}$ & 0 & $\begin{array}{l}3.627 e-05 \\
(3 / 82722)\end{array}$ & 0.016 & $\begin{array}{c}0 \\
(0 / 6429)\end{array}$ \\
\hline $1282 G>A$ & G428S & C-term & ASD241 & 2006 & M & Yes (Severe) & NO & $\begin{array}{l}\text { ADHD/mood } \\
\text { disorder } \\
\text { /Sleep } \\
\text { disturbance }\end{array}$ & Carrier & $\begin{array}{l}0.00303 \\
(1 / 330)\end{array}$ & 0 & $\begin{array}{l}0.0001256 \\
(11 / 87586)\end{array}$ & 0.044 & $\begin{array}{c}0 \\
(0 / 6634)\end{array}$ \\
\hline
\end{tabular}

AF, allele frequency; EA, East Asian; M, male; NA, not available.

a AF in our ASD cohort. All five mutations are case specific. Our Chinese ASD cohort includes 288 Chinese ASD cases with Male/Female $=246: 42$. Therefore, the adjusted allele number is 330

b $\quad$ AF in our 369 controls with Male/Female=300:69. The adjusted allele number is 438 .

c AF in East Asian population in ExAC database with Male/Female=2,016/2,311. The adjusted allele number is 6638 .

d $P$ value is calculated between AFs in Chinese ASD and ExAC by Fisher's exact test. 
Figure 1

A E18.5, DAPI

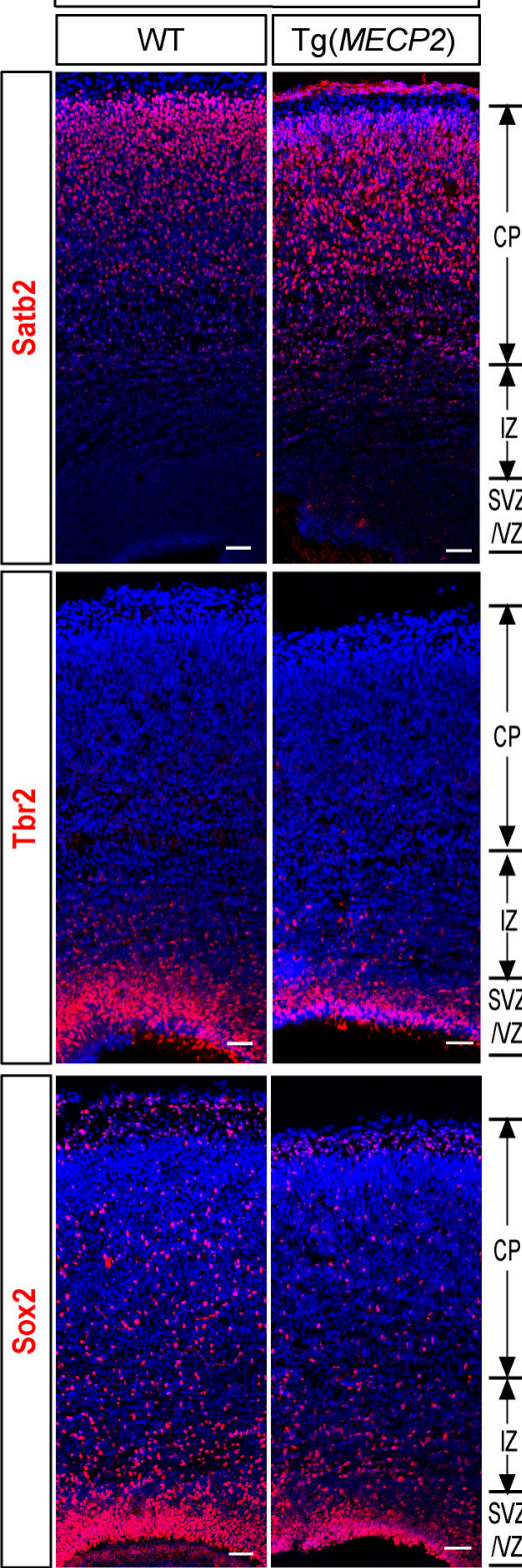

B

○WT ० $\mathrm{Tg}(M E C P 2)$

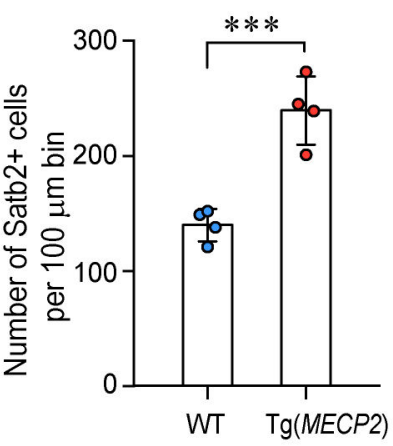

WT Tg(MECP2)

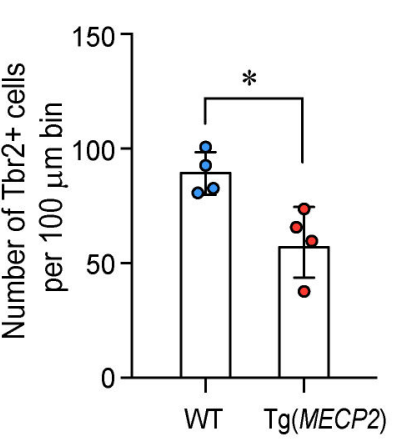

里

200

$\mathrm{CP}$

ชั

is 100

$\checkmark$ 능

1Z

$\frac{7}{\mathrm{SVZ}}$

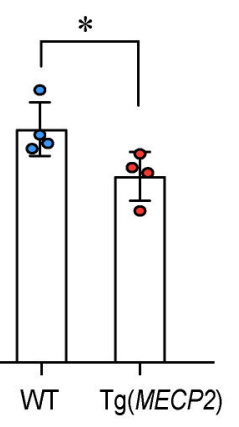

-WT ○ $\operatorname{Tg}(M E C P 2)$
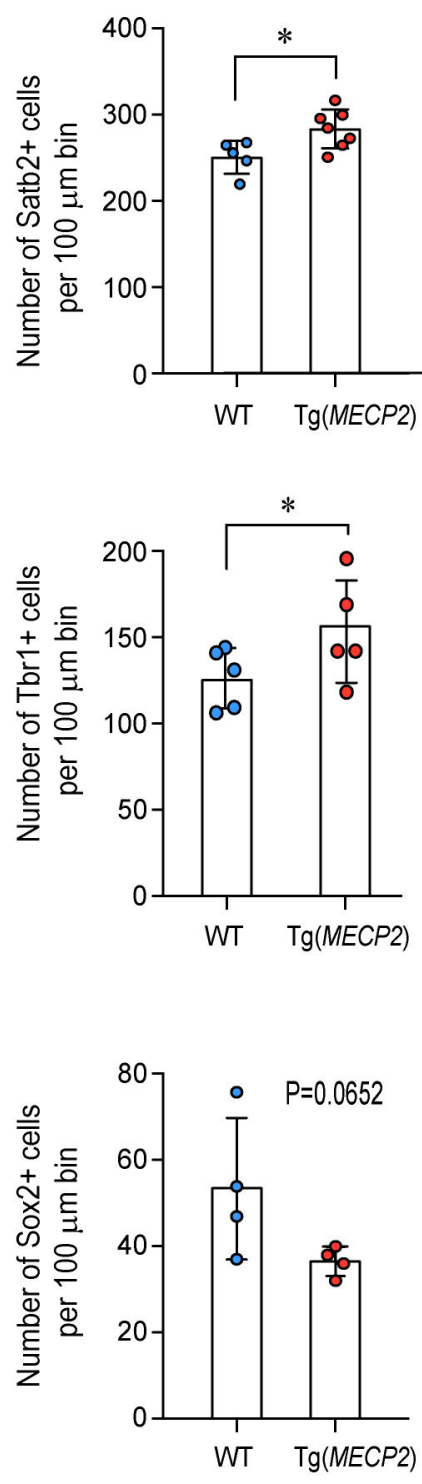
A
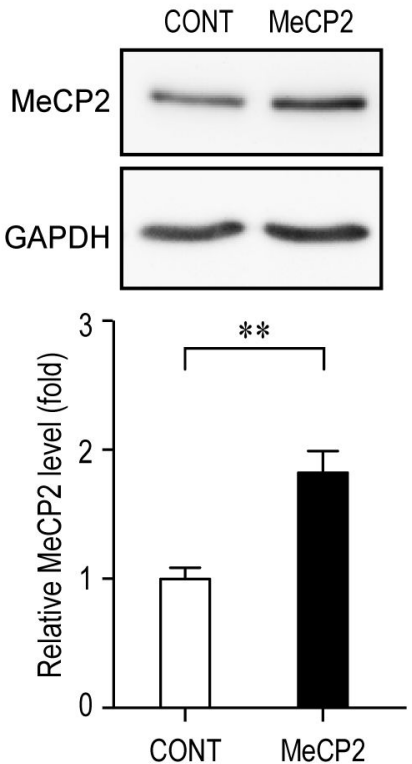

C57BL/6
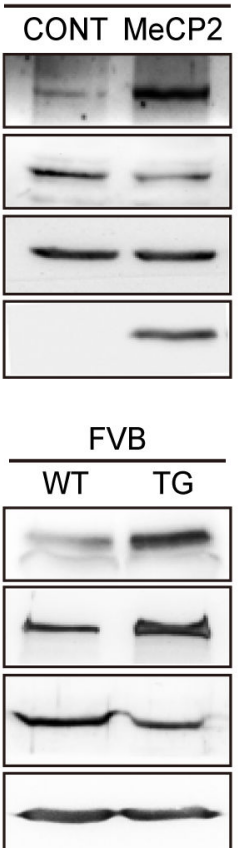

B
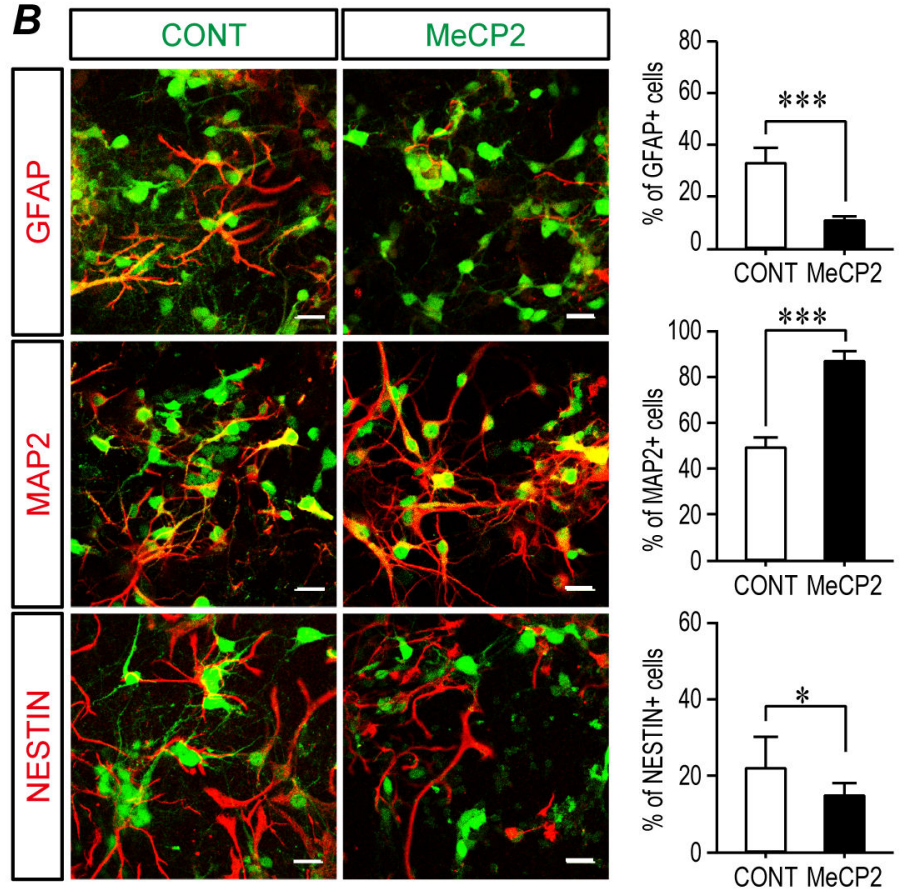

MAP2

FVB

D

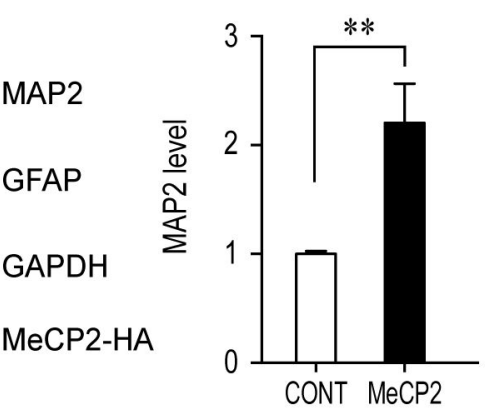

MeCP2

MAP2

GFAP

GAPDH
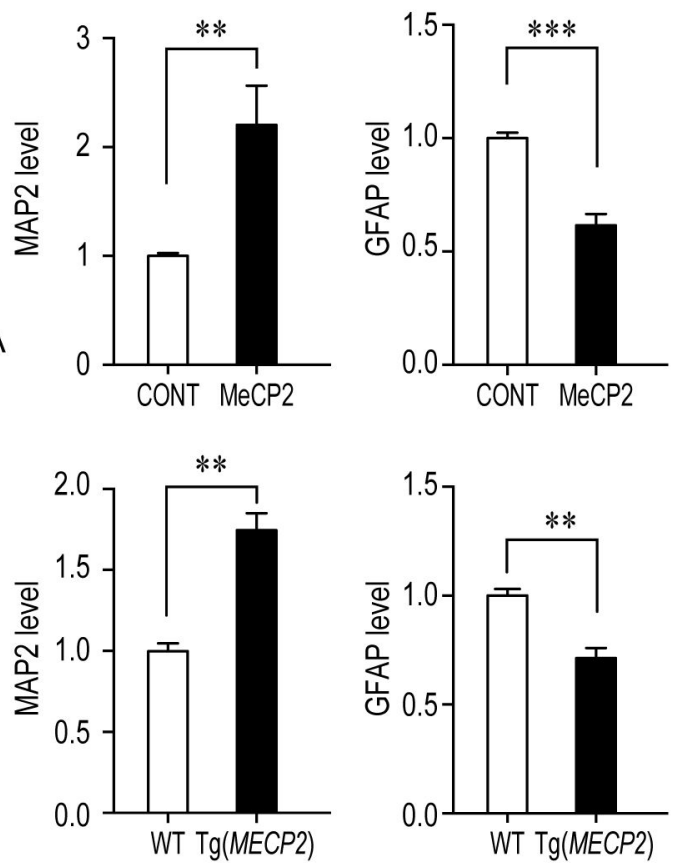

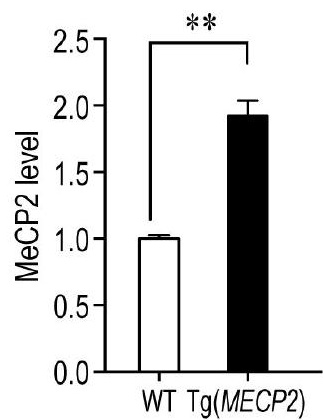


B

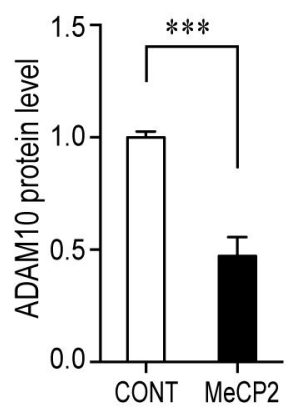

E

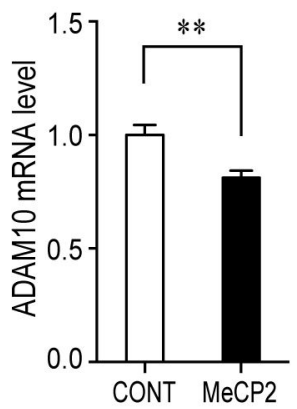

mAdam103'UTR-I 5' ...AACUUGUACAGUACGCGG-GACUUU... 3' ha10-1-Mut 5 '...AACUUGUACAGUAUGUAGUGACUUU... 3 ' hADAM10 3'UTR-I 5' ...AAACUUGUACAGUAUGUGGUGACUUU... $3^{\prime}$ IIIIIII hsa-miR-197-3p 3' CGACCCACCUCUUCCACCACUU 5'

$\boldsymbol{F}$

hsa-miR-197-3p 3' CGACCCACC-UCUUCCACCA----CUU 5' hADAM103'UTR-II 5 5....UUUGGG-GGUGGGAGGUGGAAAGGGAC... 3 hA10-II-Mut 5 '...UU_-_ AaAGGAAC... 3 ' mAdam10 3'UTR-II $5^{\prime}$...UUUUGGG-GGUGGGGGUGGGAUGGAAAAGGAAC... $3^{\prime}$

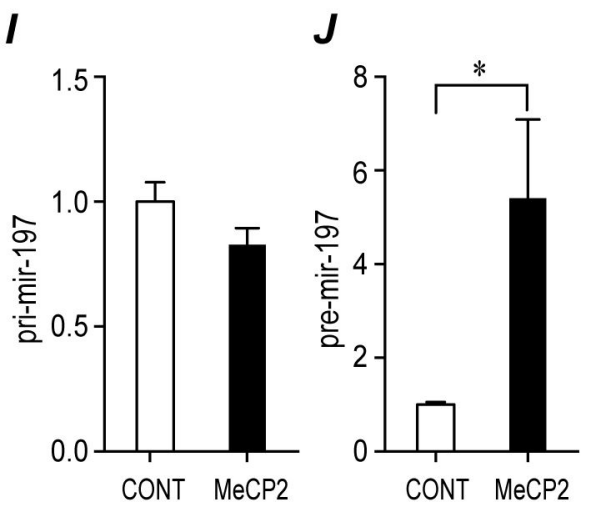

C

K

\section{D}
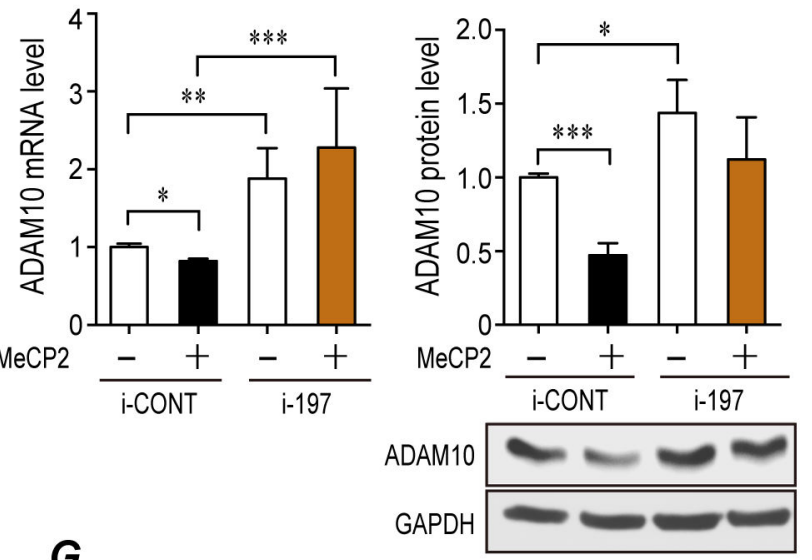

G
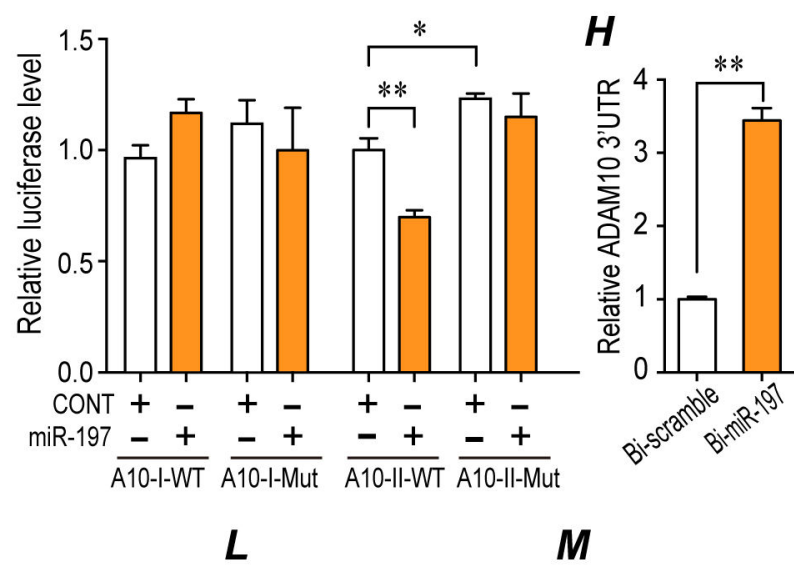

M

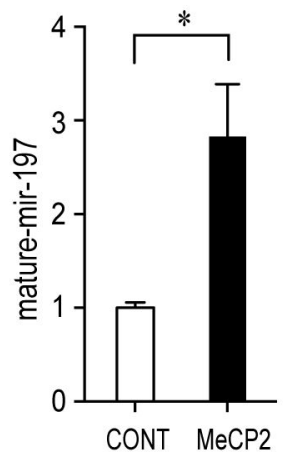

$\mathrm{MeCP2}$

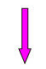

miR-197

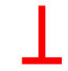

ADAM10 


\section{MECP2 duplication and mutations impair NSCs differentiation via miR-197 regulated ADAM10}

Yu-Meng Wang ${ }^{2,1, \#, ~ Y u-F a n g ~ Z h e n g 1,2,3, \#, *, ~ S i-Y u ~ Y a n g ~}{ }^{2,1}$, Zhang-Min Yang ${ }^{4}$, Lin-Na Zhang ${ }^{5}$, Yan-Qin $\mathrm{He}^{4}$, Xiao-Hong Gong ${ }^{1}$, Dong Liu ${ }^{6}$, Richard H. Finnell ${ }^{7}$, Zi-Long Qiu ${ }^{8}$, Ya-Song Dü,*, Hong-Yan Wang ${ }^{1,3,9, *}$

1. Obstetrics and Gynecology Hospital, State Key Laboratory of Genetic Engineering at School of Life Sciences, Institute of Reproduction and Development, Fudan University, Shanghai 200011, China

2. Institute of Developmental Biology \& Molecular Medicine, Fudan University, Shanghai 200433, China

3. Key Laboratory of Reproduction Regulation of NPFPC, Collaborative Innovation Center of Genetics and Development, Fudan University, Shanghai 200032, China

4. Department of Biochemistry and Molecular Biology, College of life Sciences, Shaanxi Normal University, Xi'an 710062, China

5. Shanghai Mental Health Center, Shanghai Jiaotong University, Shanghai 200030, China

6. Co-innovation Center of Neuroregeneration, Jiangsu Key Laboratory of Neuroregeneration, Nantong University, Nantong, Jiangsu 226001, China

7. Departments of Molecular and Cellular Biology and Medicine, Baylor College of Medicine, Houston, Texas 77030, USA, and Collaborative Innovation Center for Genetics \& Development, School of Life Sciences, Fudan University, Shanghai 200438, China

8. Institute of Neuroscience, Shanghai Institutes for Biological Sciences, Chinese Academy of Sciences, Shanghai, 200031, China

9. Children's Hospital of Fudan University, 399 Wanyuan Road, Shanghai 201102, China

\# These authors contribute equally.

* Corresponding authors: Hongyan Wang: wanghy@fudan.edu.cn; Yasong Du: 13501942224@163.com; Yufang Zheng: zhengyf@fudan.edu.cn

Key words: MECP2, MDS, miR-197, ADAM10, neurogenesis 


\section{Supplementary material and methods}

\section{FVB-Tg(MECP2)1Hzo/J mice Genotyping}

The genotyping of FVB- $\operatorname{Tg}(M E C P 2) 1 \mathrm{Hzo} / \mathrm{J}$ mice were determined by PCR following the protocols from Jackson Laboratory website:

https://www2.jax.org/protocolsdb/f?p=116:5:0::NO:5:P5_MASTER_PROTOCOL_ID,P5_JRS_CODE $: 14245,008679$.

\section{MeCP2 Plasmids and Lentivirus}

Human MECP2-e1 cDNA was purchased from Origene (RC202382). Each point mutant was generated by mutagenesis PCR. The lentivrus for human WT MeCP2 (HQ664), H371K (H4971), G428S (H4972), 380X (H4973), sh-MeCP2 (HQ363) and sh-control (H101) were all purchased from Obio Technology (Shanghai) Corp., Ltd.

\section{miRNA inhibitors and mimics}

Plasmids for hsa-miR-197 precursor (HmiR0013-MR04), hsa-miR-197 inhibitor (HmiR-AN0287-AM01), and scrambled negative control clone (CmiR0001-MR04) were purchased from GeneCopoeia, Inc. USA. The lentivirus for miR-197 (H4976) and miR-control (H32), i-197 (Y3496) and i-control (Y008) were all purchased from Obio Technology (Shanghai) Corp., Ltd. MiR-197 mimics (B01001) and scrambles (B04001); MiRNA inhibitors (B03001) and their negative controls (B04003) were purchased from Shanghai GenePharma Co,Ltd..

\section{Antibodies}

Antibodies used in this study were as follow: from Abcam: ADAM10 (ab1997), ADAM17 (ab13535), NICD (ab8925), GFAP (ab7260), NOTCH1 (ab27526), DLL-1 (ab76655), JAG-1 (ab7771), Nestin (ab6142), MAP2 (ab11268, ab32454), GFAP (ab7260), NeuN (ab177487), Tuj1 (class III beta tubulin, ab78078), Satb2 (ab92446), SOX2 (ab97959), TBR1 (ab31940), TBR2 (ab23345), HA (ab9110); and Alexa Fluor® 647-labeled goat anti-Rabbit (ab150079), Alexa Fluor® 647-labeled goat anti-mouse (ab150115), or Alexa Fluor® 488-labeled goat anti-Rabbit (ab150077), Alexa Fluor ${ }^{\circledR}$ 488-labeled goat anti-mouse (ab150117). Anti-MeCP2 and GAPDH antibody was 
purchased from Cell Signaling (CST, \#3456 \& \#2118S). All HRP-conjugated secondary antibodies were purchased from KangChen Bio-tech Inc., Shanghai, China.

\section{Primers}

The primers for hsa-mir-197-3p (HmiRQP0287) and the internal control snRNA U6 gene (HmiRQP9001 for human U6 and MmiRQP9002 for mouse U6) were also purchased from GeneCopoeia Inc. Primers for pri-miR-197 (4427012), pri-miR-134 (4427012), and the internal control GAPDH gene (4331182) were purchased from Applied Biosystems. Primers for pre-miR-197 (Hs_mir-197_PR_1 miScript Precursor Assay, MP00001302), pre-miR-134 (Hs_mir-134_PR_1 miScript Precursor Assay, MP00000847) and the internal control U6 gene (Hs_RNU6-2-11 miScript Primer Assay, MS00033740) were purchased from QIAGEN.

Point mutations in MECP2 were generated with the following primers.

H371R: 5'-GAGCACCACCACCATCACCGCCACTCAGAGTCCCCAAAG-3', 5'-CTTTGGGGACTCTGAGTGGCGGTGATGGTGGTGGTGCTC-3';

E394K: 5'-CCACCTGAGCCCAAGAGCTCCGAGG-3'

5'-CCTCGGAGCTCTTGGGCTCAGGTGG-3';

G428S: 5'-CACTGGAGAGCGACAGCTGCCCCAAGGAGCC-3'

5'-GGCTCCTTGGGGCAGCTGTCGCTCTCCAGTG-3'

The primers used for PCR cloning A10-II-WT are as follow.

F: 5'-CAGTCTAGACAGCTTTTGCCTTGGTTCTT-3';

R: 5'-ACTGGCCGGCCGGTCGAGCCTCCTAGCCTTGATTG-3'.

The primers used to generate A10-II-Mut are as follow:

F1: 5'-CAGTCTAGACAGCTTTTGCCTTGGTTCTT-3';

R1: 5'-AAGAAAATTGGGTTCCTTTTAATTGGA ATTTTCAGGCTTT-3';

F2: 5'-AAAGCCTGAAAATTCCAATTAAAAGGAACCCAATTTTCTT-3';

R2: 5'-ACTGGCCGGCCGGTCGAGCCTCCTAGCCTGATTG-3'.

The primers used for qRT-PCR were:

ADAM10: 5'-CCTGCCATTTCACTCTGTCATTTA-3'

5'-GTGCCCGGGCTCCTTCCTCTACTC-3';

GAPDH: 5'-ACAGCAACTCCCACTCTTCCACCT-3' 


\section{5'-TTGCTCAGTGTCCTTGCTGGGG-3'.}

The primers used for RT-PCR ADAM10 3'UTR in Biotinylated Micro-RNA Pull Down Assay were:

5'-CAGTCTAGACAGCTTTTGCCTTGGTTCTT-3';

5'-ACTGGCCGGCCGGTCGAGCCTCCTAGCC TTGATTG-3'

\section{The inhibitors of miRNA}

hsa-miR-197-3p, 5'-GCUGGGUGGAGAAGGUGGUGAA-3';

hsa-miR-221-3p, 5'-GAAACCCAGCAGACAAUGUAGCU-3';

hsa-miR-199a-5p, 5'-GAACAGGUAGUCUGAACACUGGG-3',

hsa-miR-222-3p, 5'-ACCCAGUAGCCAGAUGUAGCU-3',

hsa-miR-137, 5'-CUACGCGUAUUCUUAAGCAAUAA-3',

hsa-miR-193a-3p, 5'-ACUGGGACUUUGUAGGCCAGUU-3',

hsa-miR-184, 5'-ACCCUUAUCAGUUCUCCGUCCA-3',

hsa-miR-187-3p, 5'-CCGGCUGCAACACAAGACACGA-3'.

\section{Biotinylated Micro-RNA Pull Down Assay for Identifying miRNA Targets}

Biotinylated double stranded miRNA-197 and its scrambled control miRNA (B02003) (both Biotin-labeled at 3' end) were purchased from GenePharma. U251 cells were transfected with control miRNA and miR-197 at a final concentration of $100 \mathrm{nM}$ with RNAimax (Invitrogen ${ }^{\mathrm{TM}}$, 13778150). 24 hours post transfection, whole cell lysates were harvested in the lysis buffer (Sigma-Aldrich, I8896) supplemented with protease inhibitor (Thermo Scientific ${ }^{\mathrm{TM}}$, 78430) and RNase inhibitor (Thermo Scientific $^{T M}$, EO0381). After centrifugation, the supernatants were collected. $50 \mu$ I Streptavidin-Dyna beads (Dyna beads M-280 streptavidin) (Invitrogen ${ }^{\mathrm{TM}}$, catalog\#: 11205D) and $10 \mu \mathrm{l}$ yeast tRNA (Ambion $^{\mathrm{TM}}$, AM7119) were added to $500 \mu \mathrm{l}$ cell lysates and incubated at $4{ }^{\circ} \mathrm{C}$ overnight. The next day, $750 \mu \mathrm{l}$ Trizol and $250 \mu \mathrm{l}$ water were added per tube and kept at $-20^{\circ} \mathrm{C}$ overnight. The mixture was thawed the following day and $200 \mu \mathrm{l}$ chloroform was added and vortexed for $45 \mathrm{sec}$. The mixture was centrifuged at $4^{\circ} \mathrm{C} 18,000 \times \mathrm{g}$ for $15 \mathrm{~min}$. The upper layer was transferred to new tubes to which equal volumes of 2-propanol and $5 \mu$ glycoblue (Invitrogen ${ }^{\mathrm{TM}}$, AM9515) were added. This was incubated at RT for $10 \mathrm{~min}$ and then centrifuged at $4^{\circ} \mathrm{C} 18,000 \times \mathrm{g}$ for $15 \mathrm{~min}$. The supernatant was very carefully discarded and the pellet was washed twice with $1 \mathrm{ml}$ pre-chilled $70 \%$ ethanol. The 
pellet was air dried and resuspended in $20 \mu$ l nuclease free water. RT-PCR: $20 \mu$ RNA from biotin-labeled sample and $1 \mu \mathrm{g}$ RNA from the input sample was used for mRNA RT reactions. The miRNA enrichment calculation: Bi-miR-197 pull-down for ADAM10 3'UTR /Scramble control pull-down for ADAM10 3'UTR = X, Bi-miR-197 input/Bio-Scramble control input = Y, Fold binding = $\mathrm{X} / \mathrm{Y}$. At least three independent experiments with a minimum of three replicates each time were performed for each set. 
Figure S1. ADAM17 could not reverse MeCP2 promoted neurogenesis.

(A-E) Mouse primary NPCs isolated from E12.5 mouse embryonic cortex were transfected with either empty vector or MeCP2 expressing plasmids and cultured for $72 \mathrm{hrs}$. Cell lysates were subjected to western blot analysis for other components of NOTCH pathway except ADAM10 and NICD. $N \geqslant 5$. Representative blots are shown in $(A)$ and statistical analyses for each component was presented as follow, (B) ADAM17, (C) NOTCH1, (D) JAG1, and (E) DLL1. (F-G) Overexpression of ADAM17 could not reverse the differentiation effects of MeCP2 in electroporated embryonic brain. Fetal mouse brains were electroporated at E14.5 and the samples were collected at E18.5 for sectioning and immunostaining (F). Each condition was repeated in four different embryos from four pregnant dams $(\mathrm{N}=4)$. Representative brain sections are presented on the left side, and statistical analyses for cells in each layers are shown in (G). All statistical data represent means \pm SEM. ** $p<0.01$, *** $p<0.001$. Scale bar is $50 \mu \mathrm{m}$.

\section{Figure S2. MeCP2 overexpression promotes neurogenesis in IUE brains.}

C57BL/6 or FVB fetal mouse brains were electroporated at E14.5 and the samples were collected at E18.5 for sectioning and immunofluorescent staining with progenitor markers Sox2 and Tbr2, and neuronal markers NeuN and Tuj1 (red). DAPI (blue) was used for nuclear staining. C57BL/6 brains were electroporated with EYFP and MeCP2 expressing plasmids or control vector. FVB brains were electroporated with EYFP alone. Each condition was repeated in four different embryos from four pregnant dams $(\mathrm{N}=4)$. Representative sections were presented. Enlarged images for white boxes are presented on the bottom. Scale bar is $50 \mu \mathrm{m}$.

\section{Figure S3. MiR-197 inhibitor reversed the down-regulation effect of ADAM10 by MeCP2.}

(A) Inhibitors for miR-221, miR-222, miR-193, miR-197, miR-199, miR-137, and miR-187 were transfected into U251 cells and the levels of ADAM10 protein were examined. (B) Inhibitors for miR-221, miR-222, miR-193, miR-197, and miR-199 were co-transfected with WT MeCP2 into U251 cells, and the levels of ADAM10 protein were examined. Representative blots are shown on the top panels, and statistical analyses for ADAM10 protein are shown on the lower panels. All data represent means \pm SEM. $\mathrm{N} \geq 3, * p<0.05$, ** $p<0.01$.

\section{Figure S4. A miR-197 like small RNA could be RT-PCR amplified from E18.5 mouse cortex.}

(A) A 7mer-m8 miR-197 binding site at position 1568-1574 of human ADAM10 3'-UTR was predicted by TargetscanHuman, which is poorly conserved between human and mouse. The alignment of miR-197 to human ADAM10 3'-UTR and miR-224 to A10-I-Mut were illustrated. (B) Luciferase reporter assay showed that miR-224 but not miR-197 could down-regulate the expression of A10-I-WT. The point mutation A10-I-Mut is not sensitive to miR-224 anymore. All data represent means \pm SEM. $\mathrm{N} \geq 3$, ** $p<0.01$. (C) Fetal cortex and cerebellum from E18.5 WT and $\mathrm{Tg}(\mathrm{MECP} 2)$ FVB mice were dissected out. RNA was extracted and subjected to RT-PCR with either hsa-miR-197 
specific primer or control Rnu6 (U6 small nuclear RNA) primers. $8 \mu$ products and the DNA ladder DL500 (Takara) were run on 12\% PAGE gel without urea and stained with Gel-Red for 40min. (D) The PCR product was cloned into T-vector and sent for sequencing. The small RNA has 16 identical nucleotides to the 3 ' side of has-miR-197.

Figure S5. The miR-197 inhibitor reverses the effect of elevated MeCP2 in mouse brain.

(A) Primary NPCs from C57BL/6 mouse were transfected with MeCP2 expressing plasmid together with either inhibitor control or i-197. Cell lysates were subjected to Western blot analysis for MAP2, GFAP, and ADAM10. $\mathrm{N} \geq 4$. Representative blots are shown on the left side and statistical analyses are shown on the right side, respectively. (B) The neurogenesis stimulated in the $\mathrm{Tg}(M E C P 2)$ mouse fetal brain could be blocked by overexpressing miR-197 inhibitors (i-197). Fetal mouse brains were electroporated at E14.5 and collected at E18.5 for sectioning and immunostaining. $\mathrm{N}=4$. DAPI (blue) was used for nuclear staining. Representative staining images were presented. Scale bar is $50 \mu \mathrm{m}$. All statistical data represent means \pm SEM. *, \# $p<0.05$, **, \#\# $p<0.01, * * *, \# \# p<0.001$.

Figure S6. The sequencing data and alignment of autism-related MECP2 mutations.

(A) The MECP2 mutations in the ASD patients were confirmed by Sanger sequencing. (B) MeCP2 is a highly conserved protein. Sequence alignment of human MeCP2 e1 and e2, mouse MeCP2 e1 and $\mathrm{e} 2$, and rat MeCP2 e2 proteins by NCBI online alignment software. The mutated amino acids are highlighted in red box.

Figure S7. Exogenous expressing of MeCP2 mutants in NPCs were similar to WT-MeCP2.

Mouse primary NPCs isolated from C57BL/6 mouse E12.5 embryonic cortex were transfected with either control vector or different MeCP2 expressing plasmids and cultured for $72 \mathrm{hrs}$. Cell lysates were subjected to western blot analysis for MeCP2 protein level and GAPDH was used as a loading control. Representative blot is shown on the top panel, and statistical analysis for MeCP2 levels are shown in the bottom panel. $\mathrm{N}=3$. All statistic data represent means $\pm \mathrm{SEM}$. $* p<0.05, * * p<0.01$.

Figure S8. Three C-terminal mutant MeCP2 failed to down-regulate ADAM10 and NICD.

Human U251 cells (A) or mouse primary NPCs (B) were transfected with either WT or three C-terminal MeCP2 mutant expressing plasmids and cultured for 24hrs or $72 \mathrm{hrs}$, respectively. Cell lysates were subjected to western blot analysis for ADAM10 and NICD. Representative blots are presented on the left side and statistical analyses for cells in each layer are shown on the right side. GAPDH was used as internal control. All data represent means \pm SEM. Effects of the mutations are either compared to control (indicated with *), or WT MeCP2 (indicated with \#). N $\geq 4$, All statistical data represent means \pm SEM. * or \# $p<0.05$, ** or \#\# $p<0.01$, *** or \#\#\# $p<0.001$. 
Figure S9. MeCP2 interacts to miR-197 but not miR-134 and MeCP2 ${ }^{\mathrm{G} 428 \mathrm{~S}}$ does not bind to miR-197 anymore.

U251 cells transfected with MeCP2 expressing plasmids were also subjected to RNA-IP with MeCP2 antibody or control lgG. The levels of pri-miR-197 and pri-miR-134 co-precipitated with MeCP2 were quantified by $q R T-P C R . N \geqslant 5$, all statistical data represent means \pm SEM. $* * * p<0.001$. 


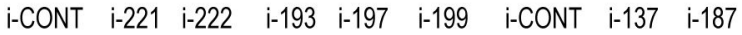
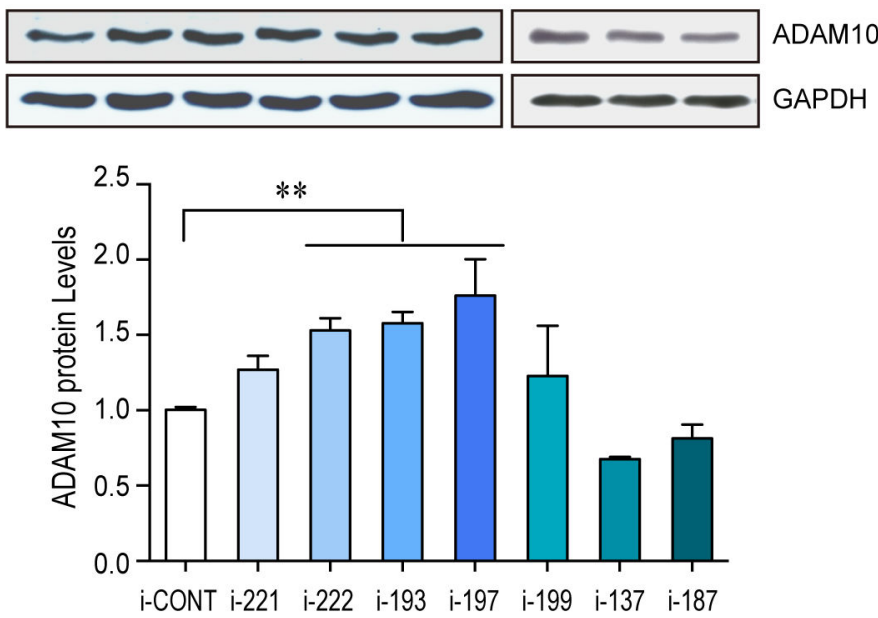

$\mathrm{MeCP} 2$
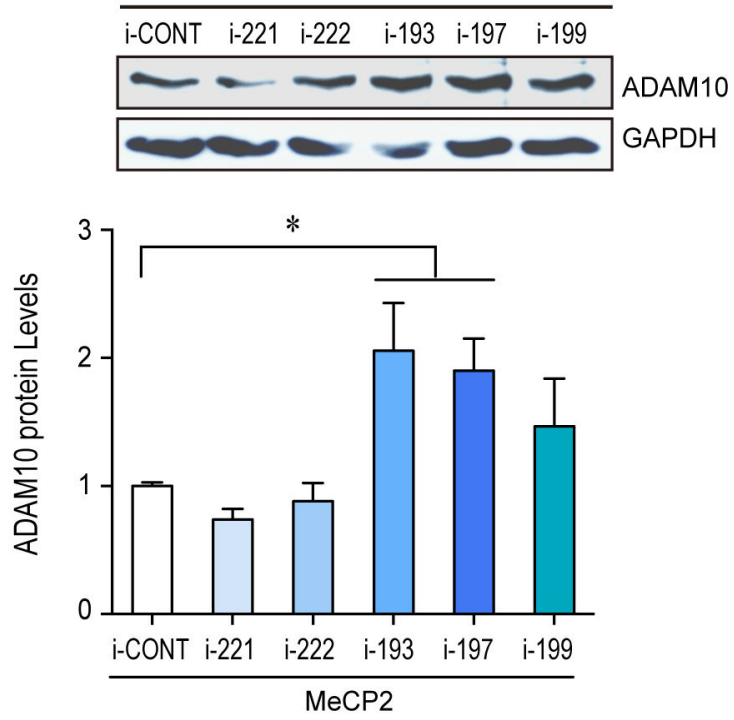
A

mAdam10 3'UTR $55^{\prime}$...AAACUUGUACAGUACGCGG-GACUUU... 3'

hA10-I-Mut $\quad 5^{\prime}$...AAACUUGUACAGUAUGUAGUGACUUU... 3'

hADAM103'UTR 5 ' ...AAACUUGUACAGUAUGUGGUGACUUU... 3' hsa-miR-197-3p 3'-CGACCCACCUCUUCCACCACUU-5'

hA10-I-Mut $\quad 5^{\prime}$...AAACUUGUACAGUAUGUAGUGACUUU... 3' IIIIIII hsa-miR-224-5p 3'-AUUUGCCUUGGUGAUCACUGAAC-5'

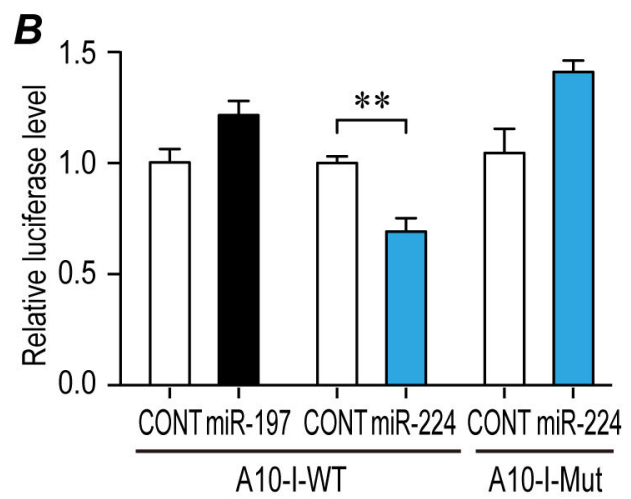

C

$\frac{\text { mir-197 }}{\frac{\text { WT }}{\text { Cortex Cereb }} \frac{\operatorname{Tg}(M E C P 2)}{\text { Cortex Cereb }}}$

$\frac{\text { U6 }}{\frac{\text { WT }}{\text { Cortex } \quad \text { Cereb }}} \frac{\operatorname{Tg}(M E C P 2)}{\text { Cortex Cereb }}$

hsa-miR-197-3p 5' UUCACCACCUUCUCCACCCAGC 3' 
ASD164, c590C>T

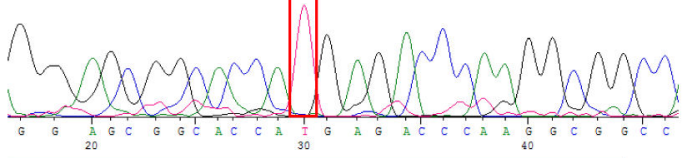

97.PRIMERL(P12504/226358.007.AB1..061

ASD97, c695G>C

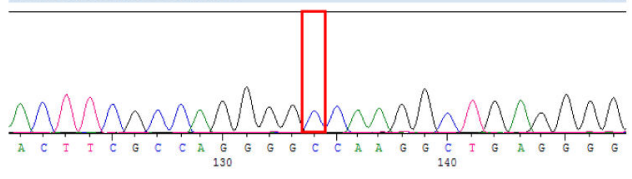

348.PRIMERRR(P12505) 226359.A03AB1. ab1

ASD348, $c 1112 A>G$

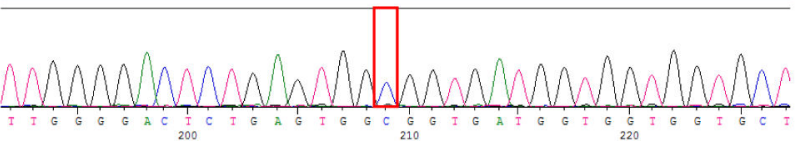

Ce: 349.PRIMERR(P12505).226360.A04.AB1.ab1

ASD349, $c 1112 A>G$

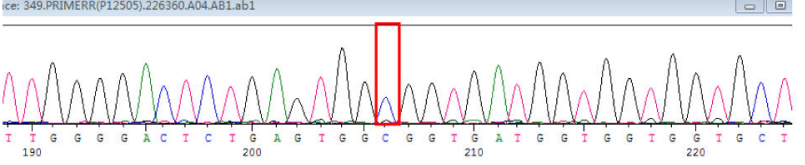

90 a o a $\mathrm{a} c=$

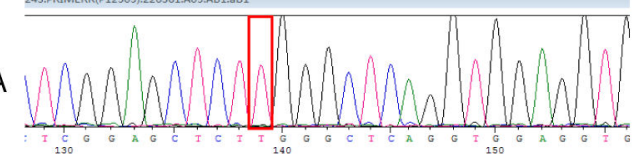

ASD243, c1180G>A

ASD241, $c 1282 G>A$

Human isoform 1 NP 004983.1

Human isoform 2 NP_001104262.1

Rat NP_073164.2

Mouse isoform 1 NP_001075448.1

Mouse isoform 2 NP_034918.1

Human isoform 1 NP 004983.1

Human isoform 2 NP_001104262.1

Rat NP_073164.2

Mouse isoform 1 NP 001075448.1

Mouse isoform 2 NP_034918.1

Human isoform 1 NP_004983.1

Human isoform 2 NP_001104262.1

Rat NP_073164.2

Mouse isoform $1 \mathrm{NP} \quad 001075448.1$

Mouse isoform 2 NP_034918.1

Human isoform 1 NP_004983.1

Human isoform 2 NP_001104262.1

Rat NP_073164.2

Mouse isoform 1 NP_001075448.1

Mouse isoform 2 NP_034918.1

Human isoform 1 NP_004983.1 Human isoform 2 NP_001104262.1 Rat NP_073164.2

Mouse isoform 1 NP_001075448.1

Mouse isoform 2 NP_034918.1

Human isoform 1 NP_004983.1 Human isoform 2 NP 001104262.1

Rat NP 073164.2

Mouse isoform 1 NP_001075448.

Mouse isoform 2 NP 034918.1

Human isoform 1 NP 004983.1

Human isoform 2 NP_001104262.1

Rat NP_073164.2

Mouse isoform $1 \mathrm{NP} 001075448.1$

Mouse isoform 2 NP_034918.1
BOY.PRIMER-RP12640).229848.E09.ABL_ab1

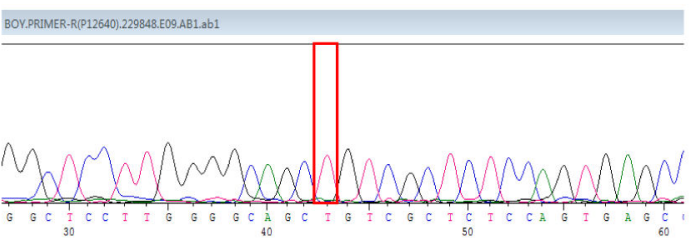

$\frac{1}{1} \frac{1}{1} \frac{1}{1} \frac{1}{1} 1$ MAAAAAAAPSG-----GGGGGEEERLEEKSEDQDLQGLKDKPLKFKKVKKDKKEEKEGKHEPVQPSAHHSAEPAEAGKAE 75 MVAGMLGLR----------------EEKSEDQDLQGLKEKPLKEKKVKKDKKEDKEGKHEPLQPSAHHSAEPAEAGKAE 63 MAAAAATAAAAAAPSGGGGGGEEERLEEKSEDQDLQGLRDKPLKFKKAKKDKKEDKEGKHEPLQPSAHHSAEPAEAGKAE 80 MVAGMLGLR------ --------EEKSEDQDLQGLRDKPLKFKKAKKDKKEDKEGKHEPLQPSAHHSAEPAEAGKAE 63

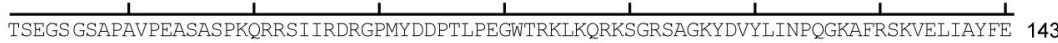
TSEGSGSAPAVPEASASPKQRRSI IRDRGPMYDDPTLPEGWTRKLKQRKSGRSAGKYDVYLINPQGKAFRSKVELIAYFE 155 TSESSGSAPAVPEASASPKQRRSI IRDRGPMYDDPTLPEGWTRKLKQRKSGRSAGKYDVYLINPQGKAERSKVELIAYFE 143 TSESSGSAPAVPEASASPKQRRSIIRDRGPMYDDPTLPEGWTRKLKQRKSGRSAGKYDVYLINPQGKAFRSKVELIAYFE 160 TSESSGSAPAVPEASASPKQRRSI IRDRGPMYDDPTLPEGWTRKLKQRKSGRSAGKYDVYLINPQGKAFRSKVELIAYFE 143

\section{T197M}

$\frac{1}{\frac{1}{K V V G D T S L D P N D F D F T V T G R G S P S R R E Q K P P K K P K S P K A P G T G R G R G R P K G S G T T R P K A A T S E G V Q V K R V L E K S P G K L L V K ~}} 223$ KVGDTSLDPNDFDFTVTGRGSPSRREQKPPKKPKSPKAPGTGRGRGRPKGSGM TRPKAATSEGVQVKRVLEKSPGKLLVK 235 KVGDTSLDPNDFDFTVTGRGSPSRREQKPPKKPKSPKAPGTGRGRGRPKGSGTGRPKAAASEGVQVKRVLEKSPGKLLVK 223 KVGDTSLDPNDFDFTVTGRGSPSRREQKPPKKPKSPKAPGTGRGRGRPKGSGT GRPKAAASEGVQVKRVLEKSPGKLVVK 240 KVGDTSLDPNDFDFTVTGRGSPSRREQKPPKKPKSPKAPGTGRGRGRPKGSGI GRPKAAASEGVQVKRVLEKSPGKLVVK 223

\section{G232A}

$\frac{1}{\text { MPFQTSPGGKAEGGGATTSTQVMVIKRPGRKRKAEADPQAIPKKRGRKPGSVVAAAAAEAKKKAVKESSIRSVQETVLPI }}$ MPFQTSP GKAEGGGATTSTQVMVIKRPGRKRKAEADPQAI PKKRGRKPGSVVAAAAAEAKKKAVKESSIRSVQETVLPI 315 MPFQASPdGKGEGGGATTSAQVMVIKRPGRKRKAEADPQAIPKKRGRKPGSVVAAAAAEAKKKAVKESSIRSVQETVLPI 303 MPFQASP GKGEGGGATTSAQVMVIKRPGRKRKAEADPQAIPKKRGRKPGSVVAAAAAEAKKKAVKESSIRSVHETVLPI 320 MPFQASPdGKGEGGGATTSAQVMVIKRPGRKRKAEADPQAIPKKRGRKPGSVVAAAAAEAKKKAVKESSIRSVHETVLPI 303

\section{H371R}

$\frac{1}{\text { KKRKTRETVSIEVKEVVKPLLVSTLGEKSGKGLKTCKSPGRKSKESSPKGRSSSASSPPKKEHHHHH H ISESPKAPVPLL }}$ KKRKTRETVSIEVKEVVKPLLVSTLGEKSGKGLKTCKSPGRKSKESSPKGRSSSASSPPKKEHHHHH H HSESPKAPVPLL 395 KKRKTRETVSIEVKEVVKPLLVSTLGEKSGKGLKTCKSPGRKSKESSPKGRSSSASSPPKKEHHHHH HAESPKAPMPLL 383 KKRKTRETVS IEVKEVVKPLLVSTLGEKSGKGLKTCKSPGRKSKESSPKGRSSSASSPPKKEHHHHHH ISESTKAPMPLL 400 KKRKTRETVSIEVKEVVKPLLVSTLGEKSGKGLKTCKSPGRKSKESSPKGRSSSASSPPKKEHHHHHH HSESTKAPMPLL 383

\begin{tabular}{|c|c|c|}
\hline E3 & & $28 \mathrm{~S}$ \\
\hline & EDPTSPPEPQDLSSSVCKEEKMPRGGSLE & $\underset{------ \text { AEKYKHRGE }_{1}}{\perp}$ \\
\hline PP & BSEDPTSPPEPQDLSSSVCKEEKMPRGGSLES & FPKEPAKTQPAVATAATA--------AEKYKHRGE 4 \\
\hline$--\mathrm{PPPPP}$ & SSEDPISPPEPQDLSSSICKEEKMPRAGSLESL & FPKEPAKTQPMVAAAATTTTTTTTTVAEKYKHRGE 4 \\
\hline-2 SPPPD & SSEDPISPP EPQDLSSSICKEEKMPRGGSLES & CPKEPAKTQPMVA--------TTTTVAEKYKHRGE 4 \\
\hline$-S P P P P$ & SEDPISPPEPQDLSSSICKEEKMPRGGSLEST & CPKEPAKTQPMVA--------TTTTVAEKYKHRGE \\
\hline
\end{tabular}

$\frac{1}{1} \frac{1}{\text { GERKDIVSSSMPRPNREEPVDSRTPVTERVS }}$

GERKDIVSSSMPRPNREEPVDSRTPVTERVS 498 GERKDIVSSSMPRPNREEPVDSRTPVTERVS 492

GERKDIVSSSMPRPNREFPVDSRTPVTERVS 501 GERKDIVSSSMPRPNREEPVDSRTPVTERVS 484 


\section{$\begin{array}{lllll}\text { CONT WT H371R E394K G428S } & \end{array}$}

$\mathrm{MeCP} 2$

GAPDH

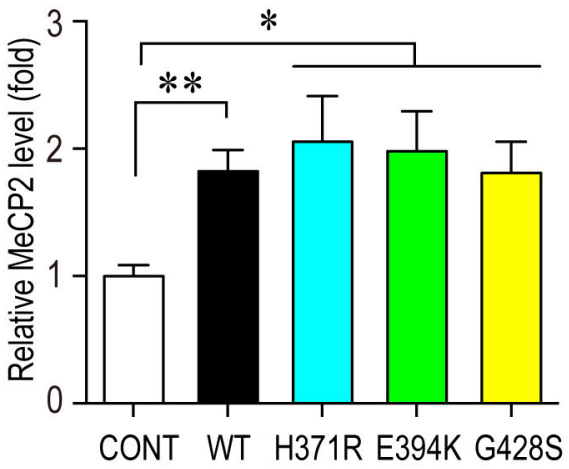




\section{A. hU251 cells}

CONT WT H371R E394K G428S
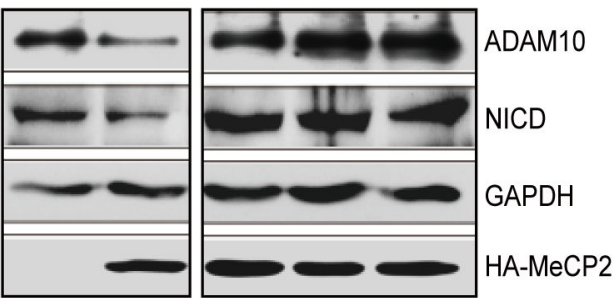

B. $m N P C s$

CONT WT H371R E394K G428S
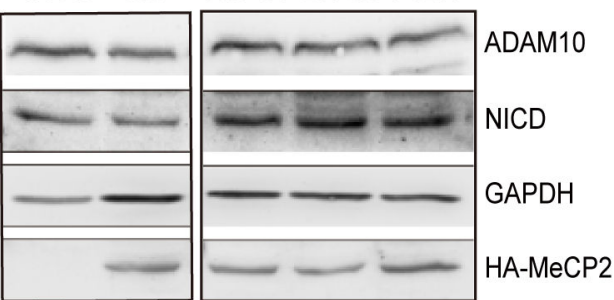
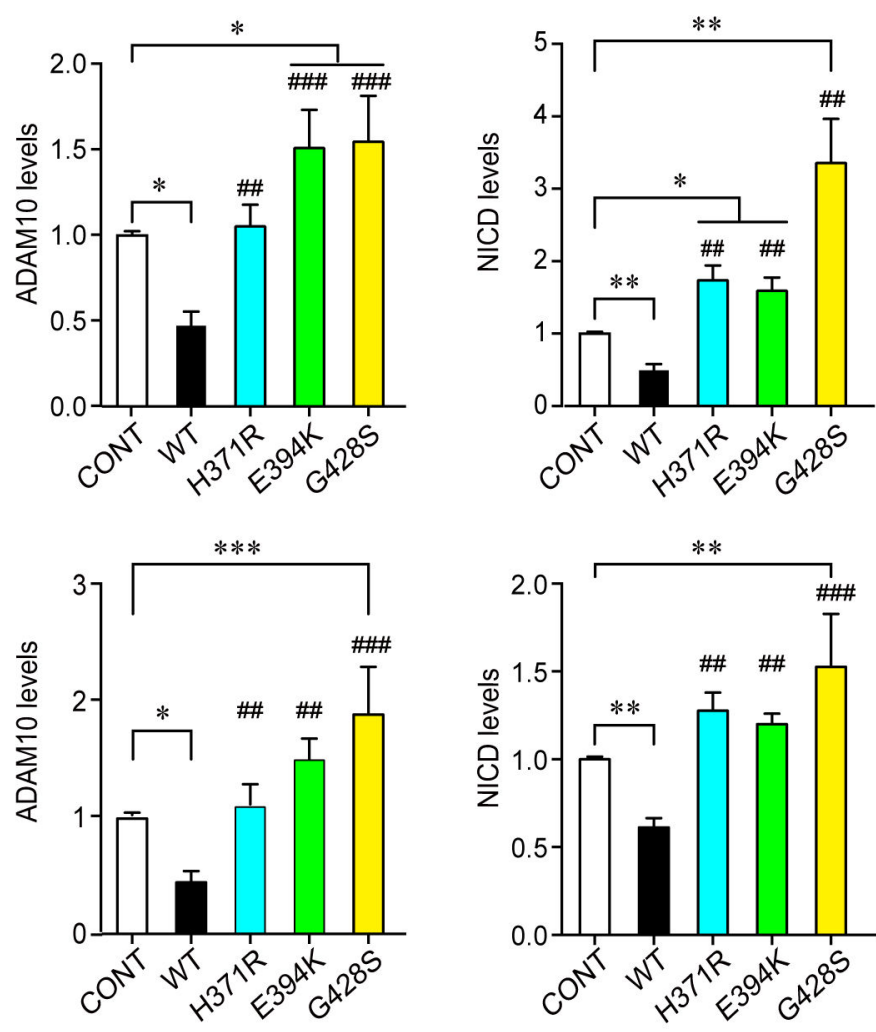


\section{Figure S9}

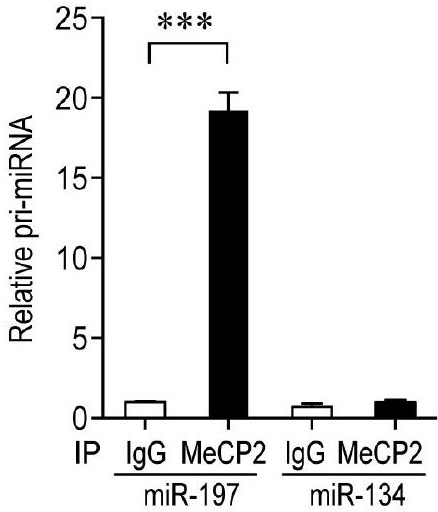

